\title{
A TEMPERATURE-INSENSITIVE GATE-CONTROLLED WEIGHTED CURRENT DIGITAL-TO-ANALOG CONVERTER
}

\author{
A Thesis \\ Presented to \\ The Graduate Faculty of The University of Akron
}

\author{
In Partial Fulfillment \\ of the Requirements for the Degree \\ Master of Science
}

Pradeep Namburu

May, 2010 


\title{
A TEMPERATURE-INSENSITIVE GATE-CONTROLLED WEIGHTED CURRENT DIGITAL-TO-ANALOG CONVERTER
}

\author{
Pradeep Namburu
}

Approved:

Thesis

Advisor

Dr. Robert Veillette

Dean of the College

Dr. George K. Haritos

Committee Member

Dr. Joan Carletta

Dean of the Graduate School

Dr. George R. Newkome

Committee Member

Date

Dr. Kye-Shin Lee

Department Chair

Dr. Alex De Abreu-Garcia 


\begin{abstract}
The current thesis presents the design of a 10-bit Digital-to-Analog Converter (DAC) to be used in a Successive Approximation Register Analog-to-Digital converter (SAR ADC). The design implements a new architecture of current-mode MOSFET DAC referred to as a gate-controlled DAC, which is found to overcome the non-linearity problems faced by the conventional drain-controlled DAC. The design also achieves temperature-insensitive operation by operating the PMOS current sources at the Zero Temperature Coefficient (ZTC) voltage, when they are turned ON.

The transistor-level circuit of a 10-bit DAC in the proposed architecture is designed and laid out, and its functionality is verified by simulations. The resulting DAC transfer characteristic is monotonic and highly linear as a result of a gate-multiplication approach to the design of the current sources. The maximum absolute error associated with the proposed design is less than $1 \mathrm{LSB}$ at $27^{\circ} \mathrm{C}$ and slightly more than $1 \mathrm{LSB}$ at $125^{\circ} \mathrm{C}$. An alternative design in which the current sources are designed using a widthcustomization approach is also simulated. This design has smaller die dimensions but is less accurate than the first design.
\end{abstract}




\section{ACKNOWLEDGEMENTS}

Firstly, I would like to thank my advisor Dr. Robert Veillette, for his guidance, support and encouragement throughout this work. I feel extremely privileged to be associated with him and shall always be grateful to him.

I would also like to thank Dr. Joan Carletta and Dr. Kye-Shin Lee for agreeing to be on my defense committee. I would like to specially thank Dr. Joan Carletta for her valuable suggestions and support.

Special thanks to Mr. Mike Ward for helping me in attaining research assistant position at The University of Akron. His suggestions, discussions and constant encouragement have helped me to get a deep insight in the field of analog/mixed signal IC design.

I take this opportunity to thank my fellow research assistants Dileep, Madhu, Matt, Nikhil and Utthej for their help and encouragement at times I needed them. Finally, I would like to thank my parents and my brother for their constant prayers and encouragement throughout my life. 


\section{TABLE OF CONTENTS}

\section{Page}

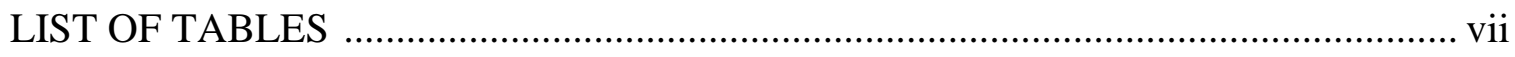

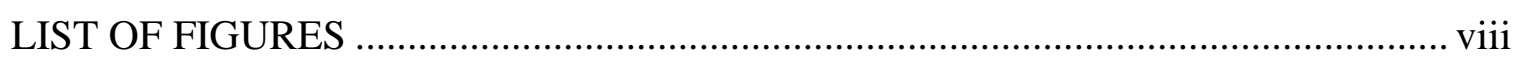

\section{CHAPTER}

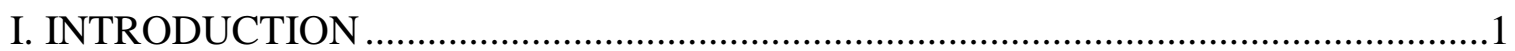

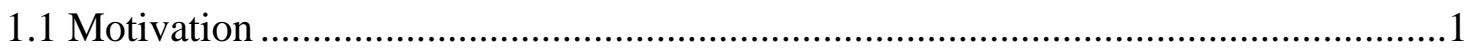

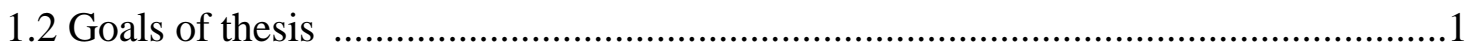

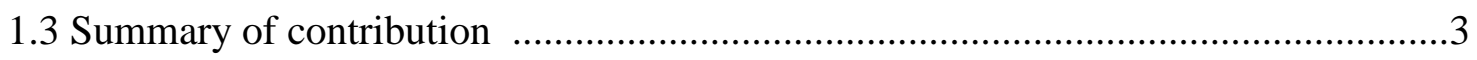

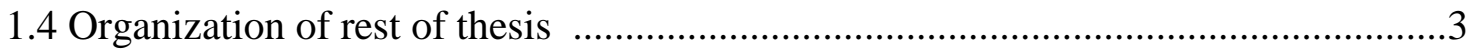

II. DIGITAL-TO-ANALOG CONVERTER BACKGROUND_.........................................

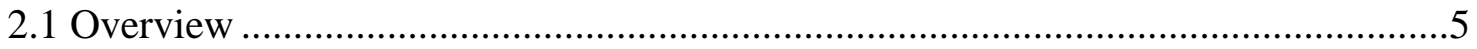

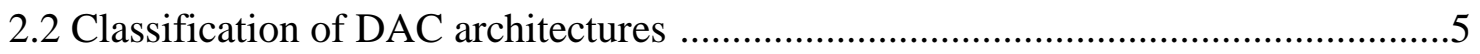

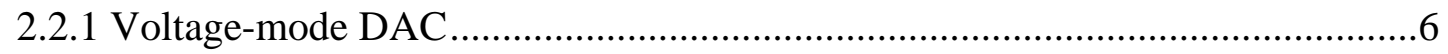

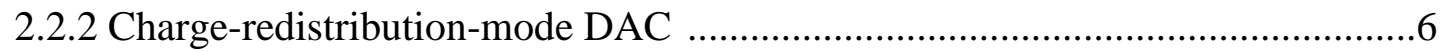

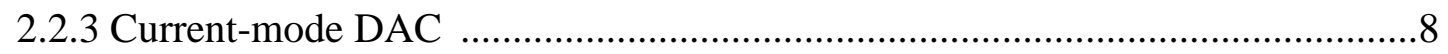

2.3 DAC performance measures ………...........................................................12

2.3.1 Integral Non-Linearity (INL) ………......................................................12

2.3.2 Differential Non-Linearity (DNL) ............................................................13

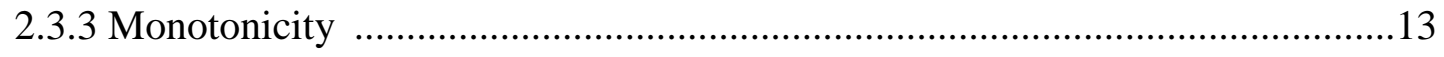




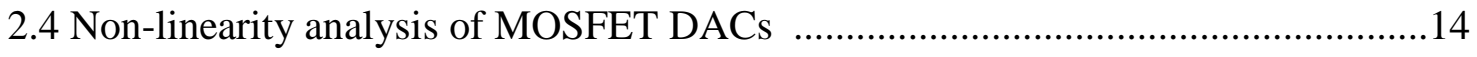

2.5 Related work in temperature-insensitive DACs ..............................................19

III. DESIGN AND ANALYSIS OF THE PROPOSED DIGITAL-TO-ANALOG CONVERTER

3.1 Design of the gate-controlled current sources ..................................................21

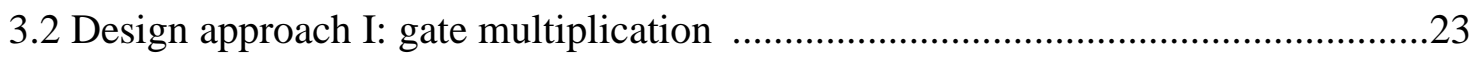

3.3 Design approach II: width customization ....................................................26

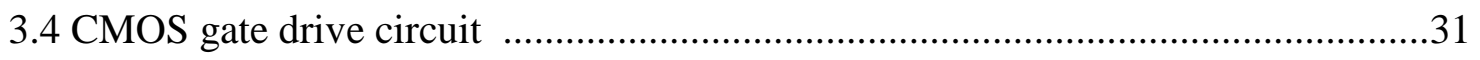

3.5 Choice of bias voltage and temperature insensitive operation ..............................31

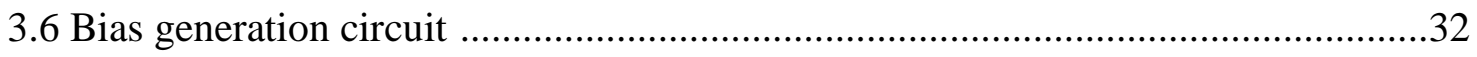

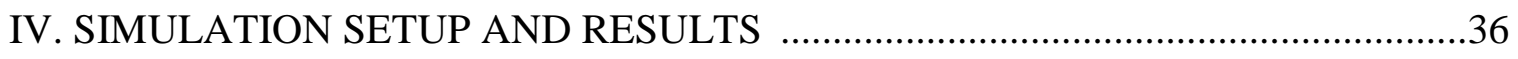

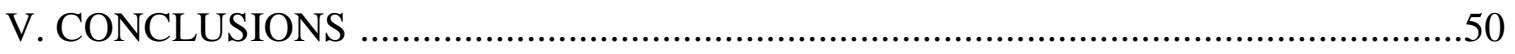

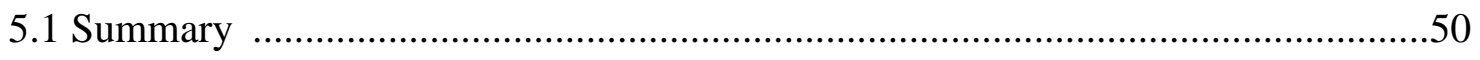

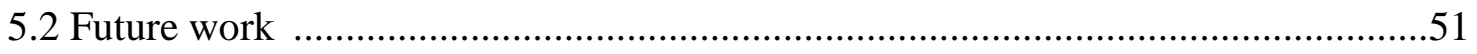

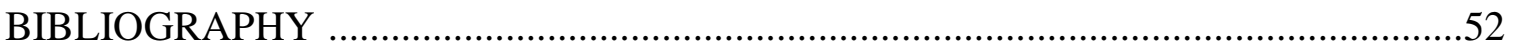




\section{LIST OF TABLES}

Table $\quad$ Page

3.1 Dimensions of the PMOS current sources obtained using gate multiplication .........24

3.2 Dimensions of the PMOS current sources obtained using width customization ........29

4.1 Currents delivered by the PMOS current sources .................................................39

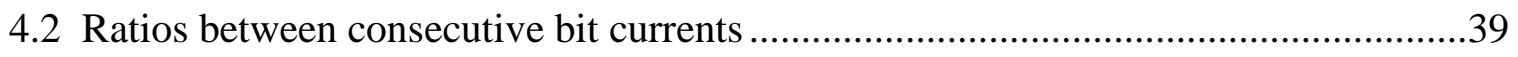

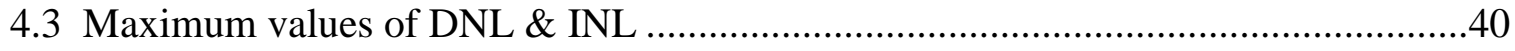




\section{LIST OF FIGURES}

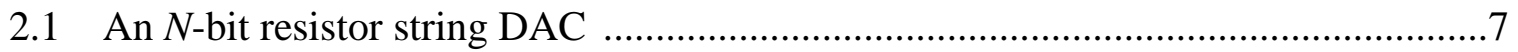

2.2 An $N$-bit charge scaling capacitive DAC ....................................................

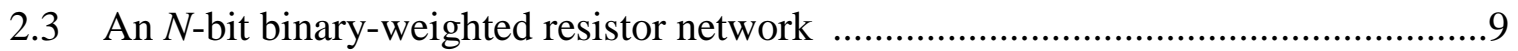

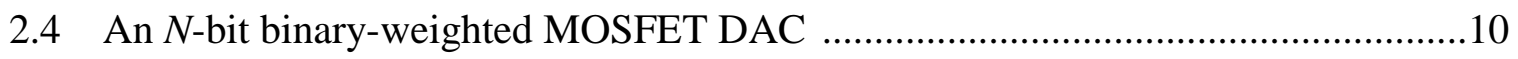

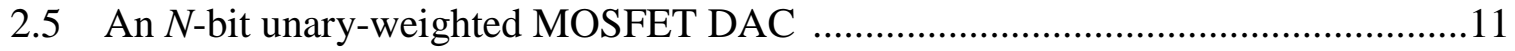

2.6 Simulation model used to obtain the transfer characteristic of a 4-bit

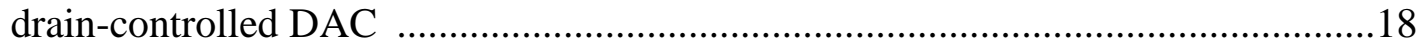

2.7 Transfer characteristic of a 4-bit drain-controlled DAC along with

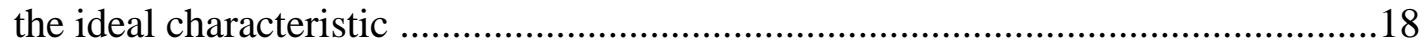

2.8 Simulation model used to obtain the transfer characteristic of a 4-bit gate-controlled DAC

2.9 Transfer characteristic of a 4-bit gate-controlled DAC along with the ideal characteristic

3.1 Implementation of one bit of the proposed DAC architecture

3.2 Block diagram of the 10-bit implementation of the proposed gate-controlled DAC

3.3 Block diagram showing the placement of the individual devices of the MSB current source, obtained using gate multiplication approach

3.4 Floor plan showing the placement of current sources of 10-bit gate-multiplied DAC 
3.5 Floor plan showing the placement of current sources of 10-bit width-customized DAC

3.6 Plot showing the I-V characteristics of a PMOS transistor ( $W=1.2 \mu \mathrm{m} ; L=5 \mu \mathrm{m})$ for different values of $V_{\text {BIAS }}$

3.7 Simulated transconductance characteristics of a PMOS transistor $(W=1.2 \mu \mathrm{m}$; $L=5 \mu \mathrm{m})$ at two different temperatures $\left(27^{\circ} \mathrm{C}\right.$ and $\left.125^{\circ} \mathrm{C}\right)$

3.8 Bias generation circuit

3.9 Plot showing the variation in the bias voltage ( $\left.V_{\mathrm{BIAS}}\right)$ with temperature .35

4.1 Test setup for the 10-bit DAC simulation .36

4.2 Output of 10-bit gate-multiplied DAC at $27^{\circ} \mathrm{C}$ and $125^{\circ} \mathrm{C}$ obtained using ideal bias voltage source

4.3 Output of 10-bit width-customized DAC at $27^{\circ} \mathrm{C}$ and $125^{\circ} \mathrm{C}$ obtained using ideal bias voltage source

4.4 Output of the 10-bit gate-multiplied DAC at $27^{\circ} \mathrm{C}$ and $125^{\circ} \mathrm{C}$ obtained using bias generation circuit

4.5 Output of the 10-bit width-customized DAC at $27^{\circ} \mathrm{C}$ and $125^{\circ} \mathrm{C}$ obtained using bias generation circuit

4.6 Transfer characteristics of 10-bit gate-multiplied DAC at $27^{\circ} \mathrm{C}$ and $125^{\circ} \mathrm{C}$ obtained using ideal bias voltage source, along with the nominal transfer characteristic

4.7 Transfer characteristics of 10-bit width-customized DAC at $27^{\circ} \mathrm{C}$ and $125^{\circ} \mathrm{C}$ obtained using ideal bias voltage source, along with the nominal transfer characteristic

4.8 Transfer characteristics of 10-bit gate-multiplied DAC at $27^{\circ} \mathrm{C}$ and $125^{\circ} \mathrm{C}$ obtained using bias generation circuit, along with the nominal transfer characteristic

4.9 Transfer characteristics of 10-bit width-customized DAC at $27^{\circ} \mathrm{C}$ and $125^{\circ} \mathrm{C}$ obtained using bias generation circuit, along with the nominal transfer characteristic

4.10 Simulated INL characteristics of 10-bit gate-multiplied DAC using ideal bias voltage source at $27^{\circ} \mathrm{C}$ and $125^{\circ} \mathrm{C}$ 
4.11 Simulated INL characteristics of 10-bit width-customized DAC using ideal bias voltage source at $27^{\circ} \mathrm{C}$ and $125^{\circ} \mathrm{C}$

4.12 Simulated INL characteristics of 10-bit gate-multiplied DAC using bias generation circuit at $27^{\circ} \mathrm{C}$ and $125^{\circ} \mathrm{C}$

4.13 Simulated INL characteristics of 10-bit width-customized DAC using bias generation circuit at $27^{\circ} \mathrm{C}$ and $125^{\circ} \mathrm{C}$

4.14 Simulated DNL characteristics of 10-bit gate-multiplied DAC using ideal bias voltage source at $27^{\circ} \mathrm{C}$ and $125^{\circ} \mathrm{C}$

4.15 Simulated DNL characteristics of 10-bit width-customized DAC using ideal bias voltage source at $27^{\circ} \mathrm{C}$ and $125^{\circ} \mathrm{C}$

4.16 Simulated DNL characteristics of 10-bit gate-multiplied DAC using bias generation circuit at $27^{\circ} \mathrm{C}$ and $125^{\circ} \mathrm{C}$

4.17 Simulated DNL characteristics of 10-bit width-customized DAC using bias generation circuit at $27^{\circ} \mathrm{C}$ and $125^{\circ} \mathrm{C}$ 


\section{CHAPTER I}

\section{INTRODUCTION}

\subsection{Motivation}

Digital-to-Analog Converters (DACs) are an integral part of most of the mixed signal systems. DACs may be used in an actuator interface or in a sensor interface such as an Analog-to-Digital Converter (ADC). The specifications of DACs largely depend on the type of application in which they are used. The specifications could be with respect to expected accuracy, operating temperature range, resolution and power dissipation. For high-temperature applications such as industrial process control and data acquisition systems, data converters may be expected to work over a wide range of temperatures ranging from $-40^{\circ} \mathrm{C}$ to $200^{\circ} \mathrm{C}$. At the same time the conversion errors associated with the design are expected to stay less than one Least Significant Bit (LSB).

\subsection{Goals of thesis}

The current thesis work focuses on building a 10-bit digital-to-analog converter to be used in a Successive Approximation Register Analog-to-Digital Converter (SAR ADC), which finds its place in the implementation of a high-temperature multi-channel sensor interface IC. The design must work over a temperature range of $27^{\circ} \mathrm{C}$ to $125^{\circ} \mathrm{C}$ without using any external circuitry. The absolute error in the DAC output must be less than $1 \mathrm{LSB}$, which is $1 / 1024$ of the full-scale output for the 10-bit DAC. 
In this thesis, we consider a current-mode MOSFET DAC in which weighted currents are added to produce a sum current that represents the digital input. A binaryweighted version of the current-mode MOSFET DAC architecture is implemented, which has the advantage that each current source is controlled by one bit of the digital input. By comparison, a unary weighted version requires complex decoding logic circuitry [1].

The various current-mode MOSFET DAC designs differ mainly in the means by which the weighted currents are switched, which forms a primary design consideration. Conventional DAC designs maintain the gate terminals of the MOSFET current sources at a constant voltage while the switches are implemented in series with the drains of the current sources; this arrangement is referred to as a drain-controlled DAC [2], [3]. A disadvantage of this architecture is that the parasitic resistances of the switches between the current sources and the sum node may introduce errors in the bit currents. In this thesis work, an alternative design is proposed in which the current sources are controlled by means of their gate voltages, while their drains are connected directly to the sum node. This design is referred to as a gate-controlled DAC. The advantage of the gate-controlled DAC is that, because there are no switches between the current sources and the sum node, the operating point of each MOS device is much more precisely known and the desired weightings of the currents are more easily achieved.

The next important goal of this thesis is to achieve temperature-insensitive operation of the proposed DAC without using any external circuitry. In this work, temperature-induced variations are expected to be minimized to a great extent by operating the PMOS current sources at the Zero Temperature Coefficient (ZTC) voltage [4], [5], when they are turned ON. 


\subsection{Summary of contribution}

A simple circuit to implement the gate-controlled version of a current-mode MOSFET DAC is introduced. The design is shown to overcome the non-linearity problems faced by the drain-controlled DAC without using high-output-resistance cascode current sources. Furthermore, the idea of biasing the current sources at the ZTC point is implemented in the proposed DAC design, and is shown to preserve linear operating characteristics over the temperature range of $27^{\circ} \mathrm{C}$ to $125^{\circ} \mathrm{C}$. The ZTC voltage that provides the bias for the current sources can be generated using an ideal voltage source or a bias generation circuit.

The transistor-level circuit of the proposed 10-bit current-mode MOSFET DAC is designed and laid out, and its functionality is verified by simulations. The resulting DAC transfer characteristic is monotonic and highly linear as a result of a gate-multiplication approach to the design of the current sources. The maximum absolute error associated with the proposed design is less than $1 \mathrm{LSB}$ at $27^{\circ} \mathrm{C}$ and slightly more than $1 \mathrm{LSB}$ at $125^{\circ} \mathrm{C}$. An alternative design in which the current sources are designed using a widthcustomization approach is also simulated. This design has smaller die dimensions but is less accurate than the first design.

\subsection{Organization of rest of thesis}

The rest of the thesis is divided into four chapters. In Chapter II, various architectures of DACs are presented along with their advantages and disadvantages, and previous work on temperature-insensitive DAC design is summarized. In Chapter III, the design of the proposed digital-to-analog converter is discussed along with the implementation methodology. In Chapter IV, the test bench setup used for the 
simulations is described followed by the simulation results. In Chapter V, the results are summarized and possible future work is discussed. 


\section{CHAPTER II}

\section{DIGITAL-TO-ANALOG CONVERTER BACKGROUND}

\subsection{Overview}

Digital-to-Analog Converters (DACs) can be implemented using different architectures, each with its own strengths and weaknesses. The performance of a DAC is characterized by various performance measures: integral non-linearity (INL), differential non-linearity (DNL), monotonicity, area of the chip and power consumption [2]. This chapter briefly discusses different types of DAC architectures, along with their advantages and disadvantages, and defines the performance measures. It also presents the non-linearity analysis of MOSFET DACs and the previous work in temperatureinsensitive DACs.

\subsection{Classification of DAC architectures}

The DAC architectures can be classified based on various factors, the most important of which is the mode of operation. On this basis, the DACs are classified as voltage-mode, charge-redistribution-mode, or current-mode DACs. In a voltage-mode DAC, elements like resistor strings are used to derive a number of different voltage levels from a reference voltage. In a charge-redistribution-mode DAC, different output levels are derived by the redistribution of charge among a set of switched capacitors. In a current-mode DAC, different output levels are obtained by dividing a major current into weighted sub-currents using elements such as resistor strings or switched current sources. 
The topology and operation of each of these three different kinds of DAC architectures are explained in the following three subsections.

\subsubsection{Voltage-mode DAC}

In a voltage-mode $\mathrm{DAC}$, a number of different voltage levels are derived from a fixed reference voltage using elements like resistor strings. Figure 2.1 shows the schematic of an $\mathrm{N}$-bit resistor string DAC, which is the simplest type of voltage-mode DAC.

In an $N$-bit resistor string DAC, $2^{N}$ identical resistors are connected in series, with the top resistor connected to a reference voltage and the bottom resistor connected to ground. The nodes between the resistors of the string have different voltages and by using binary decoding on the digital input signal one specific node can be selected as the correct analog output voltage [6].

The resistor string DAC is fast and inherently monotonic as long as the switching elements are designed correctly [6], [7]. However, the number of resistor elements becomes large when the number of bits increases; an $N$-bit DAC requires a string with $2^{N}$ resistors. With this exponential increase in the number of resistor elements, the size of high resolution DACs is prohibitively large. The other examples of voltage-mode DACs include the multiple R-string DAC, the R-2R ladder DAC and the thermometer-code DAC [6], which are generally preferred for applications with resolution less than 8 bits.

\subsubsection{Charge-redistribution-mode DAC}

A charge-redistribution-mode DAC operates on the principle of charge division, where the charge stored on an array of individually switched binary-weighted capacitors is used to perform the digital-to-analog conversion. Figure 2.2 shows the schematic of a 
charge scaling capacitive DAC, which is the simplest type of charge-redistribution-mode DAC. The most significant bit (MSB) capacitor is $2^{N-1}$ times larger than the LSB capacitor $\left(C_{0}\right)$ and the amount of charge upon each capacitor in the array is used to perform the required digital-to analog conversion. The switches are controlled by the digital input, $B=\left(b_{N-1}, \ldots, b_{0}\right)$, where $N$ is the number of bits and $b_{N-1}$ is the Most Significant Bit (MSB). After initially being discharged, the digital input switches each capacitor to either $V_{\text {ref }}$ or ground, causing the output voltage, $V_{\text {out }}$, to be a function of the voltage division between the capacitors.

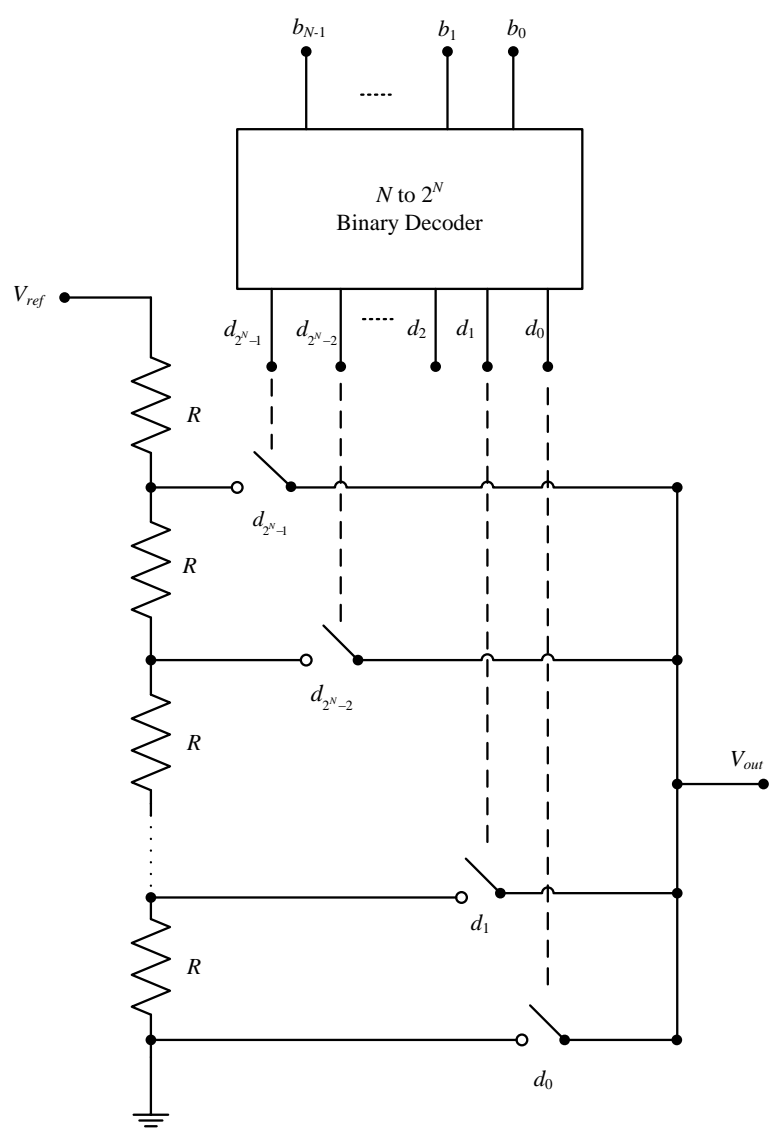

Figure 2.1 An $N$-bit resistor string DAC 
The capacitive DAC has the advantage of low static power consumption, but presents difficulties in fabrication [6]. First of all, the capacitors require a substantial area on the chip, especially when a large number of bits are required for high resolution [6], [7]. The area occupied by the capacitors can be made smaller by using a C-2C ladder network; however, the capacitors are more difficult to match than resistors would be [6], [7]. In addition to this, large transient currents are drawn from the reference voltage source during switching and also a periodic reset is required at the end of each conversion.

\subsubsection{Current-mode DAC}

In a current-mode DAC, the digital input bits are used to control a set of current sources to generate different output current levels. There are different kinds of currentmode DACs, the most prominent of which are discussed in the following sections.

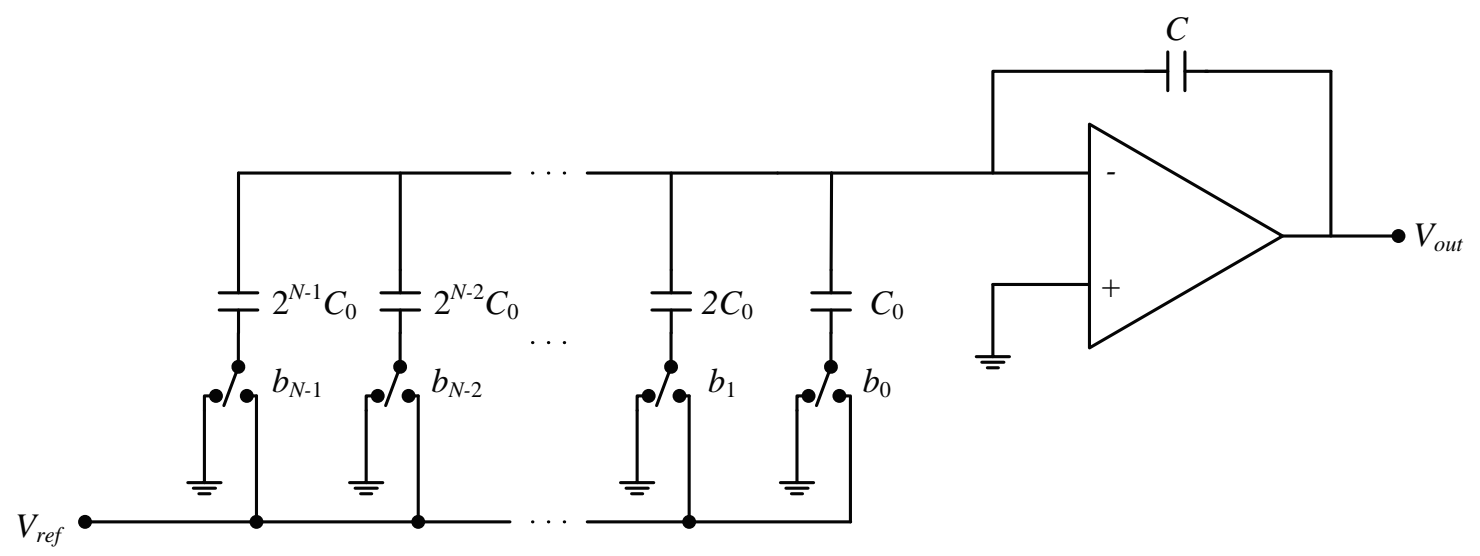

Figure 2.2 An $N$-bit charge scaling capacitive DAC 


\section{(A) Resistive DAC}

In a resistive DAC, the current division is achieved using a resistor network, which generates the required analog output. An important type of resistive network is a binary-weighted resistor network, whose architecture is shown in Figure 2.3. In this architecture, a network of $N$ binary-weighted resistors is used, which causes the digital input bits $\left(b_{N-1}, \ldots, b_{0}\right)$ to be weighted in their contribution to the output current.

The resistive DAC is simple to implement and exhibits relatively low nonlinearity errors compared to other DAC architectures [2], [6]. However, the resistor values may be affected by process variations which can lead to differences in the power consumption and in the branch currents from chip to chip [6], [7]. Even on a single chip it may be difficult to match the resistor values, and the switching components must be carefully designed not to introduce any significant parasitic resistance to the network [6], [7].

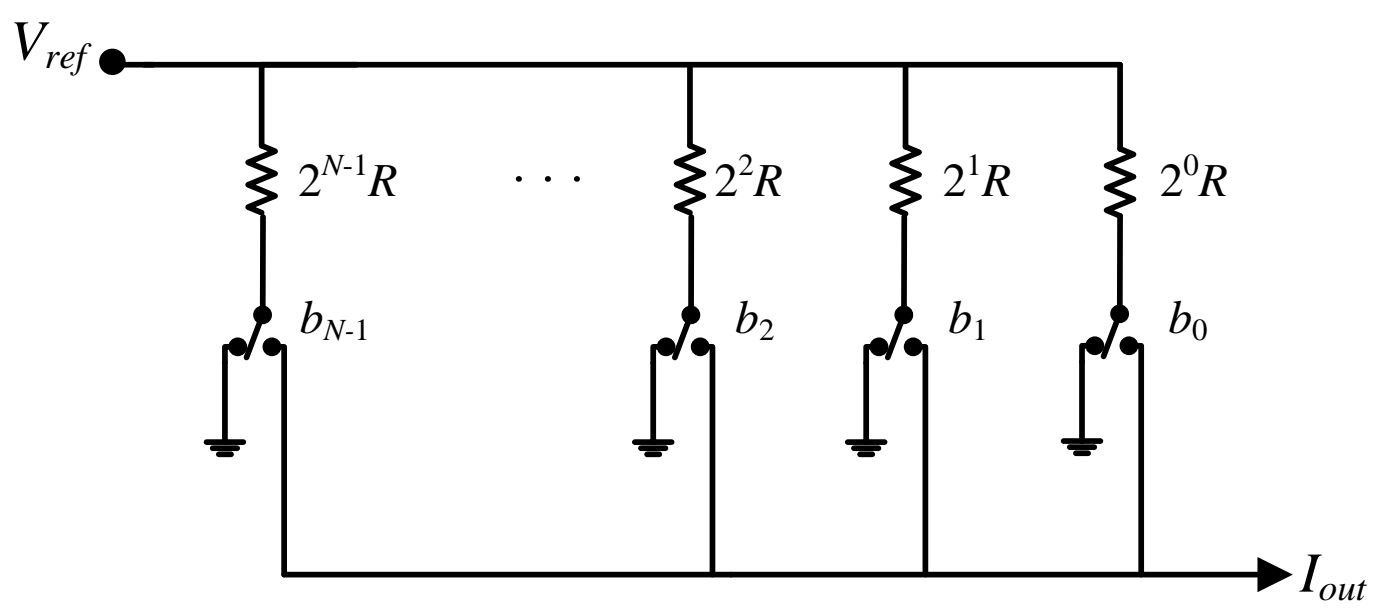

Figure 2.3 An $N$-bit binary-weighted resistor network 


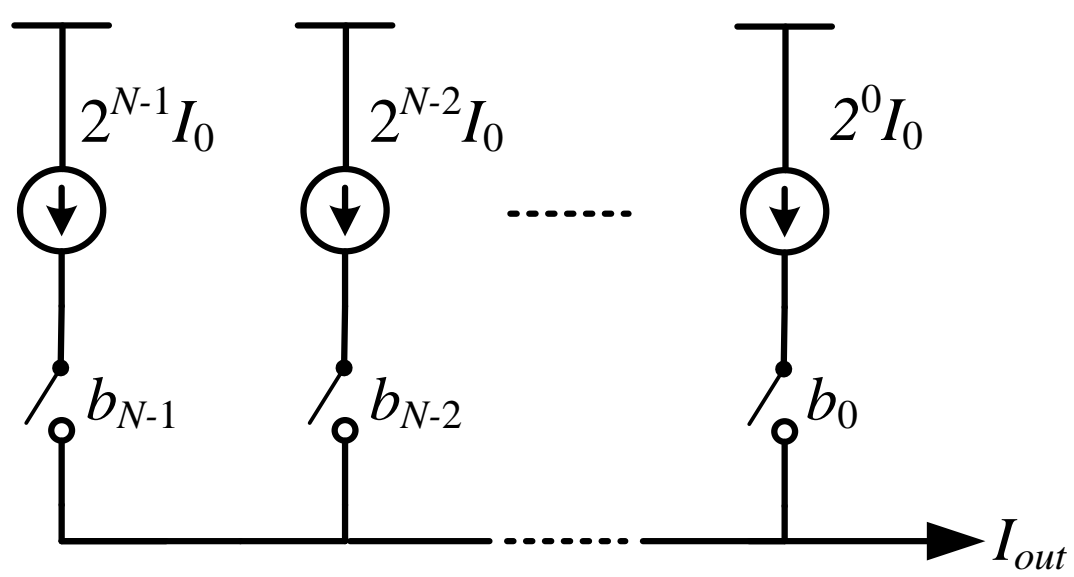

Figure 2.4 An $N$-bit binary-weighted MOSFET DAC

(B) Binary-weighted MOSFET DAC

The next important architecture is the MOSFET DAC, which is simple and relatively easy to implement, compared to resistive and capacitive DACs. The MOSFET DAC consists of an array of MOS current sources controlled by switches. Depending on the digital input, the MOS current sources that are turned ON pass their respective currents to the output node. The sum of the currents constitutes the analog representation of the digital input. The schematic of an $N$-bit binary-weighted MOSFET DAC is shown in Figure 2.4.

In a binary-weighted MOSFET DAC, the reference current $\left(I_{0}\right)$ is multiplied by powers of 2 from LSB to MSB. This requires $N$ binary-weighted current sources, each controlled by the corresponding bit of the digital input. The binary-weighted version has the advantages of simple design, small area and low power dissipation [9], [10]. However, it suffers from problems like non-monotonicity and high glitch energy [9], [10], [11]. At the same time, parasitic resistance of switches is less of a problem here 
than for the resistor network. Thus, the binary-weighted MOSFET DAC is preferred for applications in which area is a primary concern [10].

(C) Unary-weighted or thermometer-coded MOSFET DAC

An alternative implementation of a MOSFET DAC is a unary-weighted or thermometer-coded DAC. In a unary-weighted MOSFET DAC, each MOS current source produces an equal amount of current; thus $2^{N}$ current sources are required for an $\mathrm{N}$-bit digital-to-analog converter. Figure 2.5 shows the implementation of an $\mathrm{N}$-bit unary-weighted MOSFET DAC along with the binary-to-thermometer code converter with $2^{N}$ outputs.
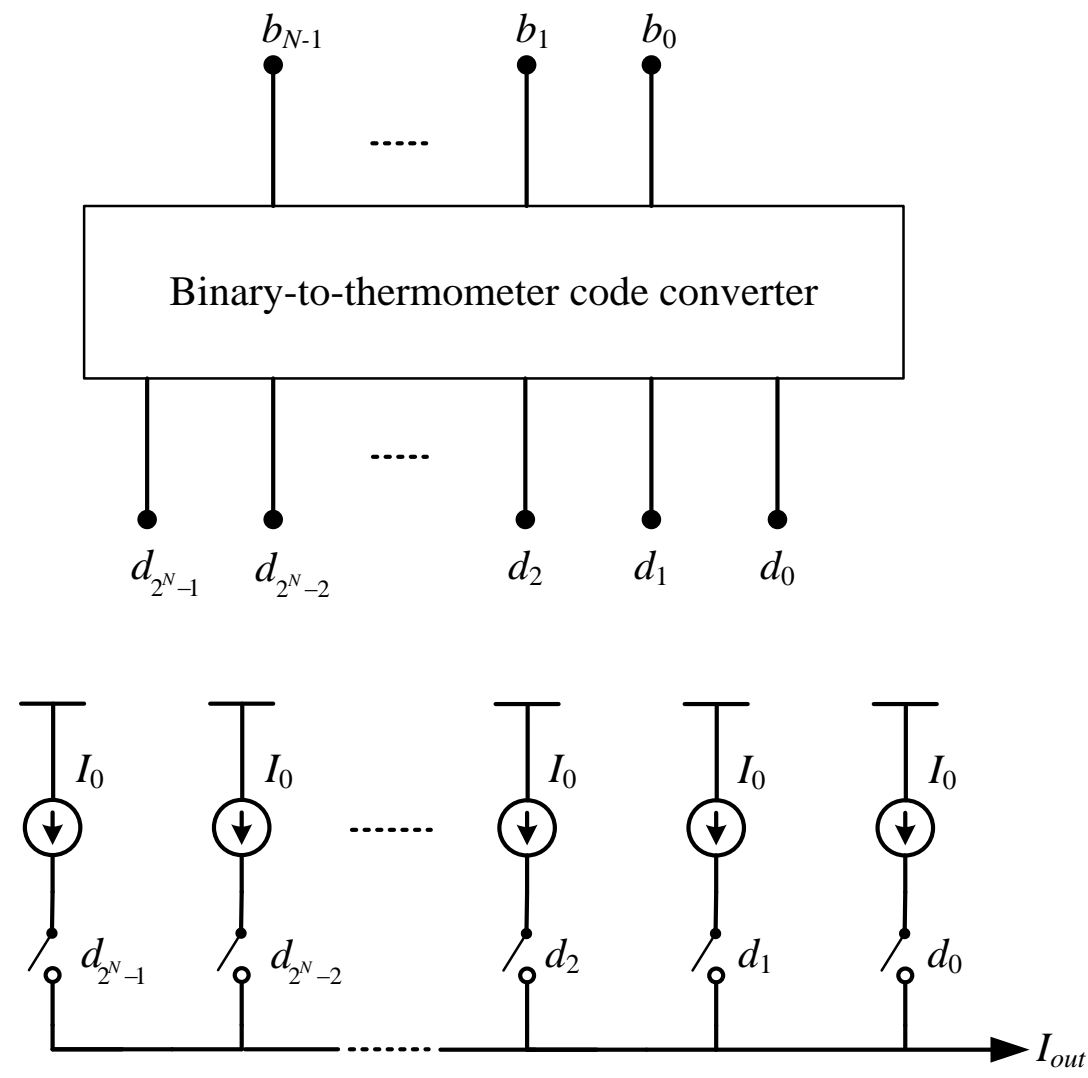

Figure 2.5 An $N$-bit unary-weighted MOSFET DAC 
The unary-weighted DAC has the benefits of monotonicity, low non-linearity errors and low glitch energy [9], [10], [11]. On the other side, the decoding logic circuit increases the design complexity, the area, and the power dissipation of the circuit and the number of switches increases exponentially with the number of bits [10], [11].

\subsection{DAC Performance Measures}

Different performance measures can be used to evaluate the quality and performance of the DACs; the most important among them are Integral Non-Linearity (INL), Differential Non-Linearity (DNL) and monotonicity.

\subsubsection{Integral Non-Linearity (INL)}

INL is defined as the deviation of the DAC transfer characteristic from an ideal one. The worst-case deviation can be expressed as a percentage of full scale or as a fraction or a multiple of an LSB. The ideal transfer characteristic can be taken as a set of $2^{N}$ discrete points on a best-fit straight line fit to the actual characteristic, or on a line drawn between the end points of the actual characteristic, or on a line through one of the endpoints of the actual characteristic assuming a given ideal slope; we call the last of these an ideal LSB extrapolation. INL is often called 'Relative Accuracy' [12].

The INL is calculated by subtracting the ideal DAC transfer characteristic from the actual DAC transfer characteristic and then dividing the results by the LSB step size. In this thesis, we choose the ideal LSB extrapolation approach, matching the ideal characteristic at the lower end to obtain the INL values. The equation used to calculate INL for the $j^{\text {th }}$ digital input code is:

$$
\mathrm{INL}_{j}=\left[I_{j}-\left(j \times I_{\mathrm{LSB}}+I_{\mathrm{ZS}}\right)\right] /\left[I_{\mathrm{LSB}}\right],
$$


where $I_{j}$ is the measured output current corresponding to the $j^{\text {th }}$ digital input code, $I_{\mathrm{LSB}}$ is the current ideally generated when the LSB current source alone is turned ON and $I_{\mathrm{ZS}}$ is the zero-scale current value.

\subsubsection{Differential Non-Linearity (DNL)}

DNL is defined as the error in the difference between the measured output responses for successive DAC codes. DNL shows how much the step between two adjacent code analog values deviates from the ideal one-LSB step. The worst-case DNL can be expressed in terms of fractions of LSB or as a percentage of full scale. An ideal DAC response would have analog output values exactly one-LSB apart, which corresponds to a zero DNL error [12], [13].

The DNL is calculated by computing the error in each step size and then dividing the results by the average LSB step size. The equation used to calculate DNL for the $j^{\text {th }}$ digital input code is:

$$
\mathrm{DNL}_{j}=\left[\left(I_{j}-I_{j-1}\right) / I_{\mathrm{LSB}}\right]-1,
$$

where $I_{j}$ is the measured output current corresponding to the $j^{\text {th }}$ digital input code and $I_{\mathrm{LSB}}$ is the current ideally generated when the LSB device alone is turned ON.

\subsubsection{Monotonicity}

A monotonic function has a slope whose sign does not change. A monotonic DAC has an output that changes in the same direction (or at worst remains constant) for each increase in the input code. A non-monotonic DAC has a maximum absolute DNL greater than 1 LSB [12], [13]. 


\subsection{Non-linearity analysis of MOSFET DACs}

Non-linearity is one of the major issues with the design of MOSFET DACs and it largely depends on the architecture chosen. The parasitic resistances associated with the DAC architecture contribute to the non-linearity. In the case of a drain-controlled DAC, the resistances of the switches present between the current sources and the sum node may cause significant errors in the currents delivered by the sources over process variations and hence introduce a significant non-linearity in the DAC transfer characteristic.

This situation can be illustrated using an example of a 4-bit binary-weighted MOSFET DAC, a model of which is shown in Figure 2.6. The bit current sources are not ideal and thus each one has an output resistance $R_{i}$ connected in parallel. Each of the current sources is connected to the sum node by means of a switch, whose resistance is modeled as a resistor $R_{d}$. The model is constructed to include the effects of changes in the bit currents as well as in the output resistances of the current sources and the resistances of the switches. The voltages $V_{1}$ and $V_{2}$ are held constant at zero potential, so that the model accounts only for the incremental currents in the resistors.

The current sources are assumed to be made from MOSFETs of a given fixed length, with their widths scaled by powers of two for the different bits. The current characteristics of the larger MOSFETs have higher variations with the drain-to-source voltage; thus, the incremental output resistances of the larger MOSFETs will be

proportionally smaller. Therefore, the ideal currents and output resistances are obtained using the relations

$$
\begin{gathered}
I_{k}=I_{0} \times 2^{-k}, k=0,1,2,3 \\
R_{i k}=R_{i 0} \times 2^{k}, k=0,1,2,3
\end{gathered}
$$


where $I_{0}$ is the ideal MSB current and $R_{i 0}$ is the incremental output resistance of the MSB current source.

The switches connecting the current sources to the sum node are assumed to be made from MOSFETs sized in proportion to the bit currents that they carry. The ideal switch resistances are therefore expressed as

$$
R_{d k}=R_{d 0} \times 2^{k}, k=0,1,2,3
$$

where $R_{d 0}$ is the incremental resistance of the switch between the MSB current source and the sum node. The nominal parasitic resistances $R_{d}$ of the switches affect the currents flowing in the circuit as current division occurs between those resistances and the internal source resistances $R_{i}$. If the same proportions are maintained between $R_{d}$ and $R_{i}$ for each bit, their effect on the DAC output current is linear over the entire range of the transfer characteristic.

Now, let us consider the case where process variations are introduced in the model of the 4-bit binary-weighted MOSFET DAC. To represent the effect of process variations, perturbations are introduced in the magnitudes of the currents delivered by the sources and the parasitic resistances of the sources and switches. To accomplish the simulation, two random vectors $\operatorname{rand}_{1}$ and $\mathrm{rand}_{2}$ whose elements are uniformly distributed between -1 and +1 are generated. The parasitic resistances and the currents delivered by the sources are modified using the relations

$$
\begin{aligned}
I_{k} & =I_{0} \times 2^{-k} \times\left(1+\operatorname{rand}_{1}(k) \times 0.2\right), k=0,1,2,3 \\
R_{i k} & =R_{i 0} \times 2^{k} \times\left(1+\operatorname{rand}_{1}(k) \times 0.2\right), k=0,1,2,3 \\
R_{d k} & =R_{d 0} \times 2^{k} \times\left(1+\operatorname{rand}_{2}(k) \times 0.2\right), k=0,1,2,3 .
\end{aligned}
$$


The factor of 0.2 in each expression ensures that the perturbations are no greater than $20 \%$ of the nominal values. Such a large variation is allowed to make the non-linearity clearly visible in the result. Using the modified values, the transfer characteristic of the drain-controlled DAC is obtained from simulations for the 16 possible digital input combinations $\left(2^{4}=16\right)$. The results for one particular random choice of the perturbation vectors are shown in Figure 2.7. It can be seen that a significant non-linearity is introduced in the DAC transfer characteristic.

The non-linearity observed in Figure 2.7 can be partially attributed to the presence of the switches between the current sources and the sum node in the model. The nonlinearity may be reduced by making the resistances of the switches smaller or the output resistances of the sources larger. A standard approach is to use cascode current sources to obtain high source resistances. In this thesis, an alternative approach is introduced, in which no switches are connected between the current sources and the sum node. For the proposed design, referred to as a gate-controlled DAC, the switch resistances are eliminated; thus, the source resistances are less critical to the DAC performance.

The model of a 4-bit binary-weighted MOSFET gate-controlled DAC is shown in Figure 2.8. Again the current sources are not ideal and thus each has an internal resistance $R_{i}$ connected in parallel. The voltages $V_{1}$ and $V_{2}$ are held constant at zero potential. In this case, as the voltage across the non-ideal current sources remains constant, the parallel source resistances do not have any effect on the DAC characteristic. The perturbations introduced in the magnitudes of the currents delivered by the sources alone have an effect on the DAC characteristic. 
Again the process variations are modeled by introducing perturbations in the currents delivered by the sources and the parasitic resistances of the sources in the same way as for the drain-controlled DAC model. To accomplish the simulation, the same perturbation vector rand $_{1}$ is used as for the drain-controlled DAC model. The transfer characteristic of the gate-controlled DAC is obtained from simulations for the 16 possible digital input combinations $\left(2^{4}=16\right)$. The results are shown in Figure 2.9. It can be seen that the non-linearity introduced by the modeled process variations is less severe for the gate-controlled DAC model than for the drain-controlled DAC model. This can be attributed to the absence of the switches between the current sources and the sum node.

Considering the implementation requirements of the MOSFET DACs, one can envision a trade-off in choosing between a standard drain-controlled design and the proposed gate-controlled design. For the drain-controlled design, to avoid the need to use large switches between the current sources and the sum node, cascode current sources must be used to overcome the effects of the parasitic switch resistances. By contrast, for the gate-controlled design, a single MOSFET can be used for each current source. This reduces the number of MOSFETs required in the design, and may reduce the overall area of the circuit. Moreover, it allows a straightforward design approach for temperature insensitivity by the use of the Zero Temperature Coefficient (ZTC) operating point of the current-source MOSFETs, as discussed later in this thesis. The disadvantage of the gatecontrolled design is that the interface from the digital input bits requires larger drivers. This is because the input bits must drive the current sources directly in the gate-controlled design, whereas they only must drive the switches in the drain-controlled design. 


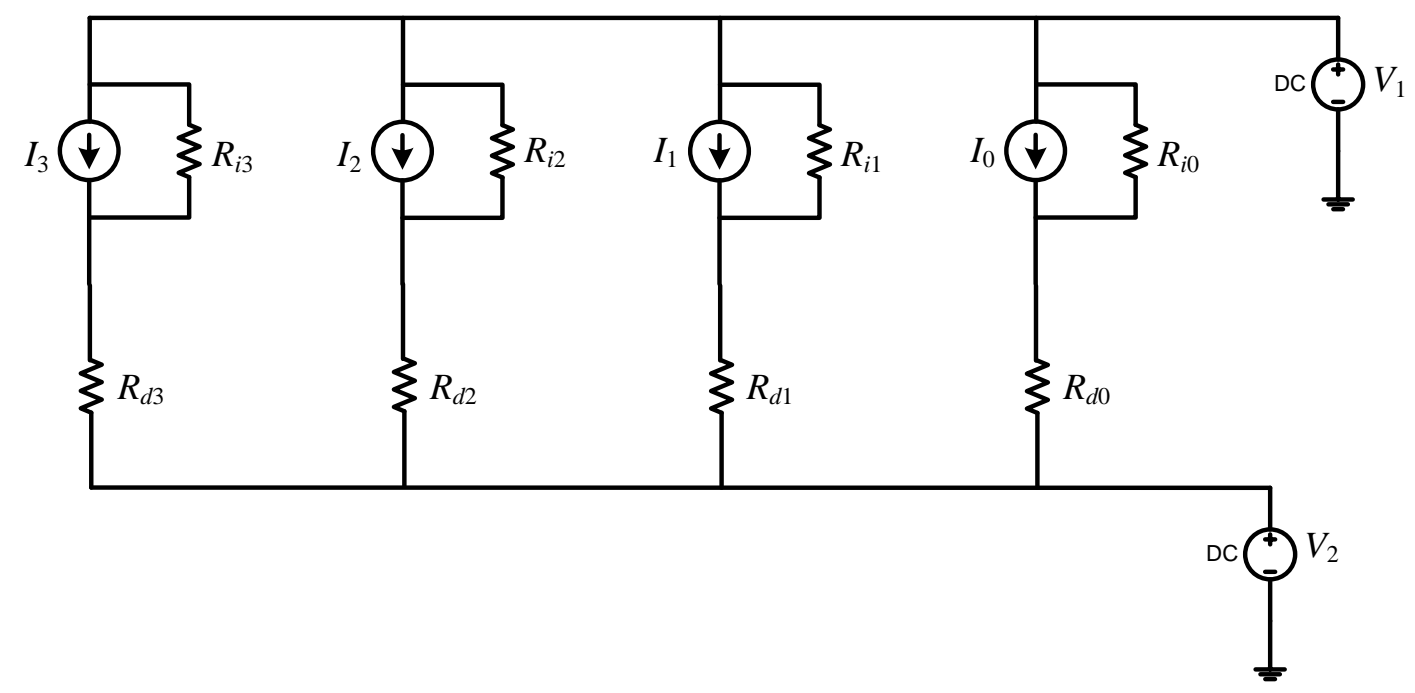

Figure 2.6 Simulation model used to obtain the transfer characteristic of a 4-bit draincontrolled DAC

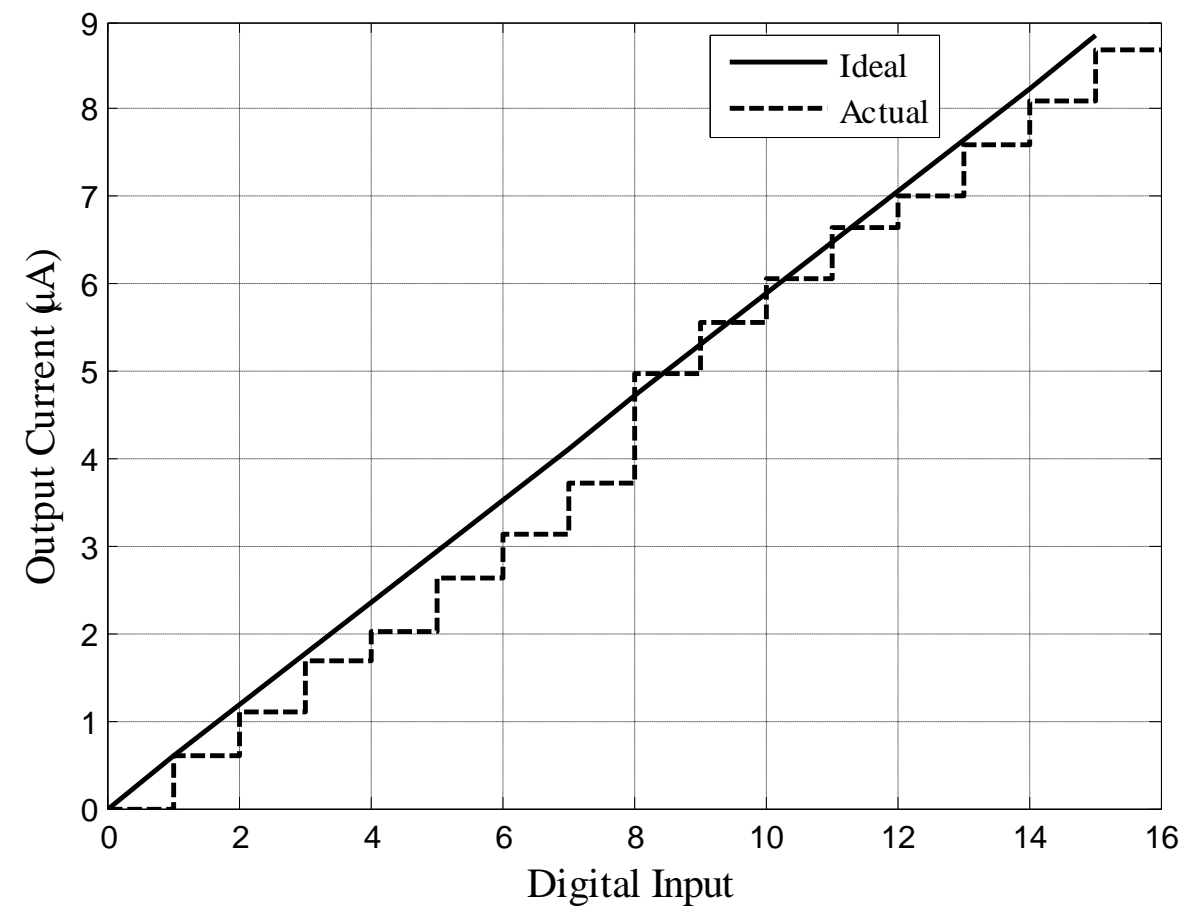

Figure 2.7 Transfer characteristic of a 4-bit drain-controlled DAC along with the ideal characteristic 


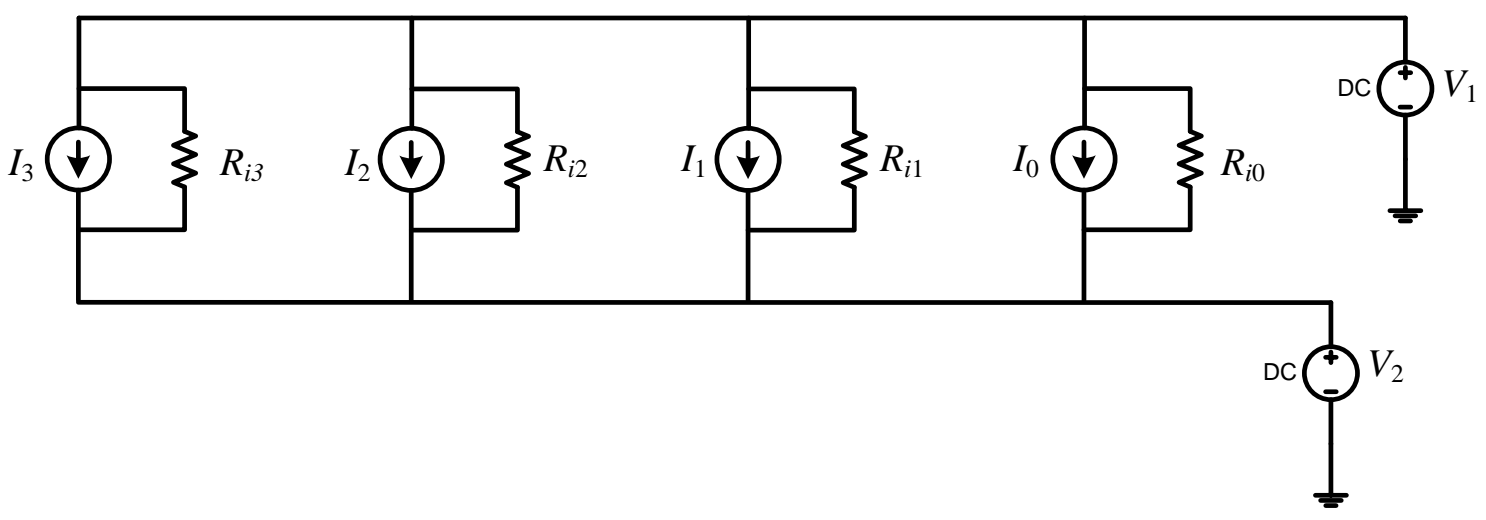

Figure 2.8 Simulation model used to obtain the transfer characteristic of a 4-bit gate-controlled DAC

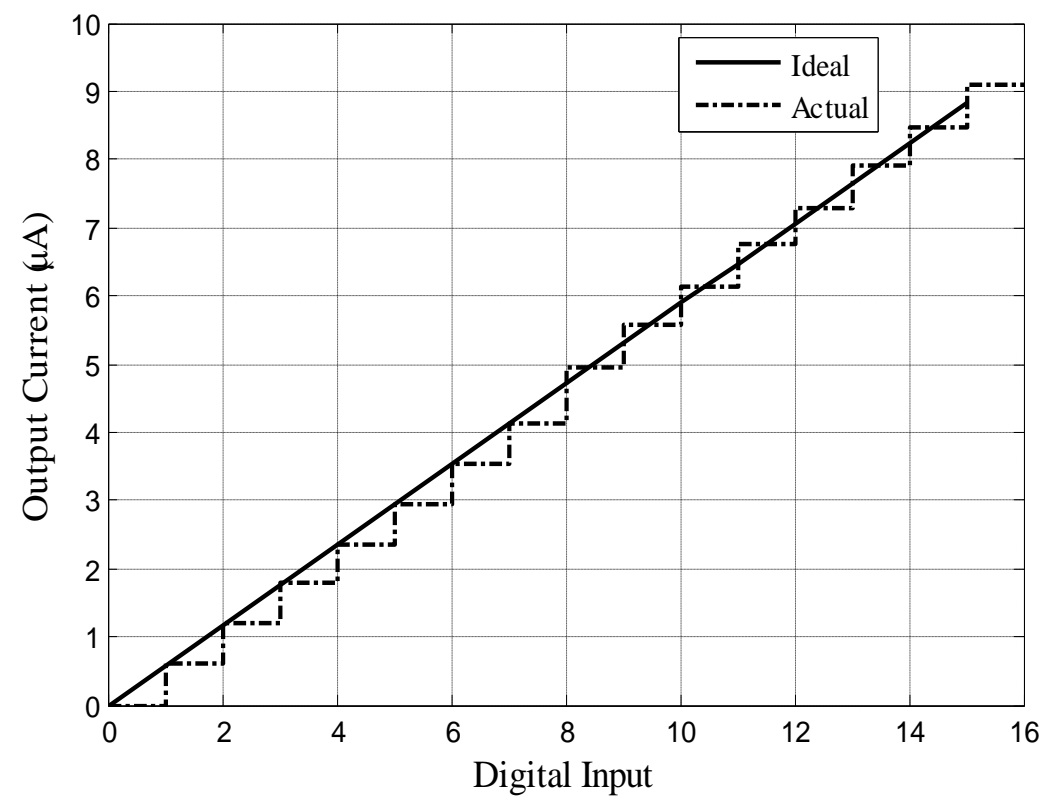

Figure 2.9 Transfer characteristic of a 4-bit gate-controlled DAC along with the ideal characteristic

\subsection{Related work in temperature-insensitive DACs}

An important design consideration of DACs is to make them work over a wide range of operating temperatures. The techniques developed to obtain temperatureindependent operation of a DAC are discussed in this section. Reilly [14] used an additional temperature compensation circuit that compensates for temperature-induced 
variations in a PWM (Pulse Width Modulation) DAC but consumes considerable power and occupies a substantial area on the chip.

Shah and Collins [15] designed a MOSFET current source choosing the bias voltage around the temperature-invariant region, which is referred to as the ZTC (Zero Temperature Coefficient) voltage [4], [5] to produce an adjustable output current which is constant over a wide range of temperatures. This approach proved simple and fairly effective, but the proposed current source was not implemented in a digital-to-analog converter. In this thesis, temperature-insensitive operation of the DAC is achieved by operating the current sources at the ZTC voltage when they are turned ON. Thus, temperature-insensitive operation is achieved without using external circuitry. This idea of biasing the current sources of a DAC at the ZTC voltage has not been found in the literature.

In this thesis, the bias $\left(V_{\mathrm{BIAS}}\right)$ required for the PMOS current sources is first simulated as an ideal voltage source. As an alternative, $V_{\mathrm{BIAS}}$ is also implemented using a simple on-chip bias generation circuit, whose area and power consumption are insignificant compared to the overall chip requirements.

The design of the proposed current-mode MOSFET DAC and the method of achieving temperature-insensitive operation by operating the PMOS current sources at the ZTC voltage are explained in detail in the following chapter. 


\section{CHAPTER III}

\section{DESIGN AND ANALYSIS OF THE PROPOSED DIGITAL-TO-ANALOG CONVERTER}

The gate-controlled weighted-current DAC presented in this thesis is designed to provide temperature-insensitive operation over a temperature range from $27^{\circ} \mathrm{C}$ to $125^{\circ} \mathrm{C}$. The proposed DAC is laid out assuming 0.5- $\mu \mathrm{m}$ Silicon-On-Insulator (SOI) technology with approximate die dimensions of $495 \mu \mathrm{m} \times 135 \mu \mathrm{m}$. An alternative design and layout with reduced die dimensions of $150 \mu \mathrm{m} \times 92 \mu \mathrm{m}$ is also given. In Chapter IV, a comparison is made between the two designs taking into consideration several criteria such as bit current ratios, INL and DNL.

\subsection{Design of the gate-controlled current sources}

The proposed implementation of the $i^{\text {th }}$ current source of the DAC, which is controlled through a CMOS driver, is shown in Figure 3.1. The current source is implemented with a PMOS transistor M1 $i$, which is designed to produce the desired binary-weighted bit current when driven to saturation. The current source (M1 $i)$ is controlled by the digital input bit $\left(b_{i}\right)$ through a CMOS driver, built from a PMOS-NMOS pair $(\mathrm{M} 2 i-\mathrm{M} 3 i)$

When the digital input $\left(b_{i}\right)$ is LOW, the PMOS transistor of the driver (M2i) is turned $\mathrm{ON}$ and the gate voltage of the PMOS current source (M1i) is driven to $V_{D D}$, 
turning M1i OFF. Conversely, when the digital input $\left(b_{i}\right)$ is HIGH, the NMOS transistor of the driver (M3i) is turned $\mathrm{ON}$ and the gate voltage of the PMOS current source (M1i) is driven to $V_{B I A S}$, which is low enough to drive M1 $i$ to saturation.

A block diagram of the 10-bit implementation of the proposed gate-controlled DAC is shown in Figure 3.2. The current sources are designed in such a way that the currents produced by them are successively scaled by higher powers of two as we move from the Least Significant Bit (LSB) to the Most Significant Bit (MSB). The currents are then added together at the sum node (at the bottom of the circuit) to produce the sum current $I_{\text {OUT }}$. Hence, the sum current can be expressed in terms of the input bits and the reference current as

$$
I_{\text {OUT }}=2^{9} I_{0} \cdot b_{9}+\ldots+2^{1} I_{0} \cdot b_{1}+2^{0} I_{0} \cdot b_{0} .
$$

Thus, the resultant sum current $I_{\text {OUT }}$ is proportional to the value of the digital input.

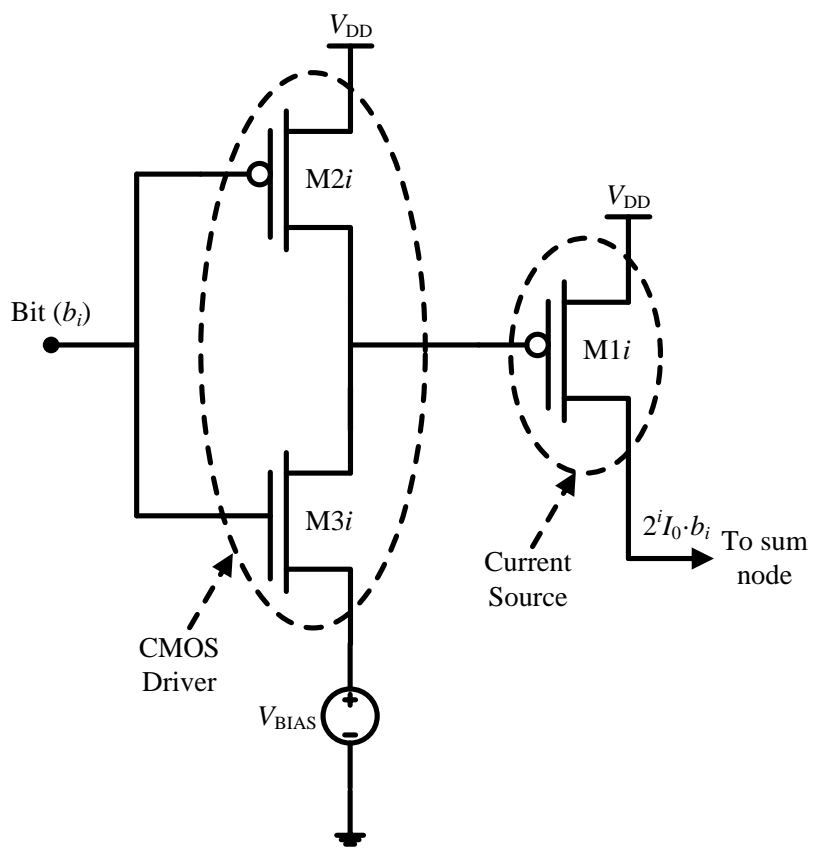

Figure 3.1 Implementation of one bit of the proposed DAC architecture 


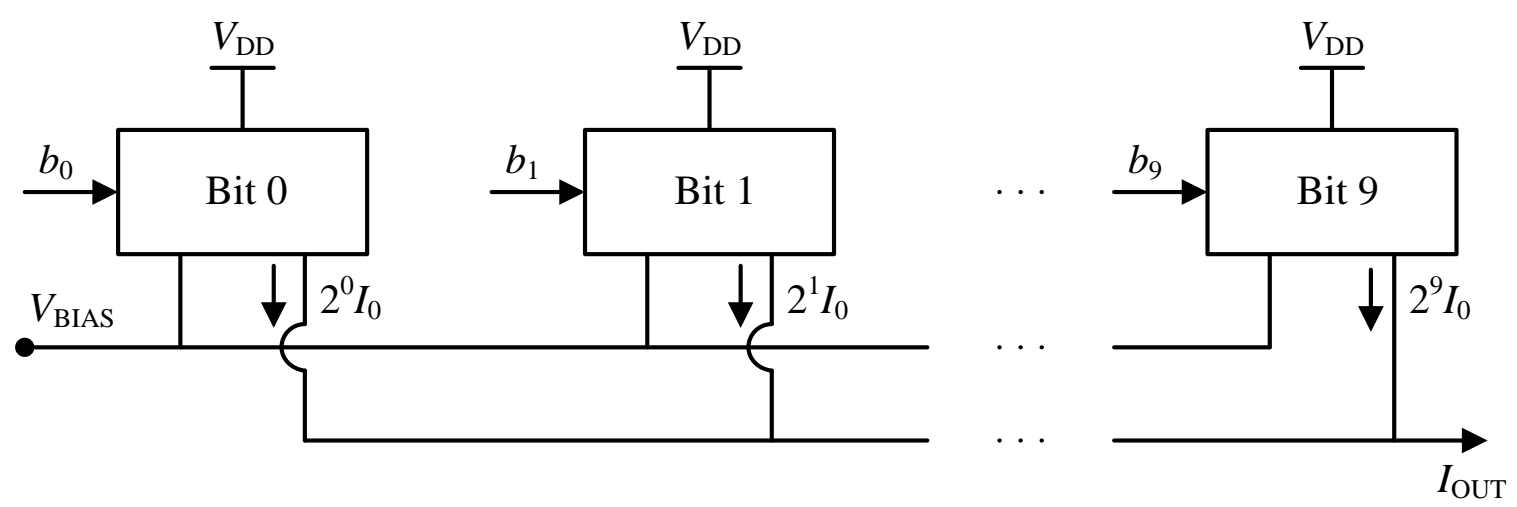

Figure 3.2 Block diagram of the 10-bit implementation of the proposed gate-controlled DAC

The binary scaling of the current sources mentioned above is an essential feature of the DAC design. Two different approaches to achieve accurate scaling are discussed in the following sections.

\subsection{Design approach I: gate multiplication}

The simplest way to achieve the binary weightings of the bit currents is just to multiply the number of PMOS devices used to implement each current source by the appropriate power of two. That is, the LSB current source is made of a single PMOS device, the next is made of two identical PMOS devices, the next is made of four, and so on. The MSB current source is made of $2^{N-1}$ identical PMOS devices. The sizes of the PMOS current sources obtained from this design approach are summarized in Table 3.1. The LSB current source is obtained using a single PMOS device with dimensions of ( $W$ $\times L)=(1.2 \mu \mathrm{m} \times 5 \mu \mathrm{m})$. The MSB current source is obtained by connecting 512 identical PMOS devices in parallel, each with dimensions of $(W \times L)=(1.2 \mu \mathrm{m} \times 5 \mu \mathrm{m})$, giving a total drawn width of $614.4 \mu \mathrm{m}$. 
Table 3.1 Dimensions of the PMOS current sources obtained using gate multiplication

\begin{tabular}{|c|c|c|c|}
\hline Current Source & $(W(\mu \mathrm{m}) \times L(\mu \mathrm{m}))$ & No. of Devices & Total Drawn Width $(\mu \mathrm{m})$ \\
\hline M10 $(\mathrm{LSB})$ & $(1.2 \times 5)$ & 1 & 1.2 \\
\hline M11 & $(1.2 \times 5)$ & 2 & 4.8 \\
\hline M12 & $(1.2 \times 5)$ & 4 & 9.6 \\
\hline M13 & $(1.2 \times 5)$ & 8 & 19.2 \\
\hline M14 & $(1.2 \times 5)$ & 16 & 38.4 \\
\hline M15 & $(1.2 \times 5)$ & 32 & 76.8 \\
\hline M16 & $(1.2 \times 5)$ & 64 & 153.6 \\
\hline M17 & $(1.2 \times 5)$ & 128 & 307.2 \\
\hline M18 & $(1.2 \times 5)$ & 256 & 614.4 \\
\hline M19 $($ MSB $)$ & $(1.2 \times 5)$ & 512 & \\
\hline
\end{tabular}

The proportions of the bit currents are expected to be very accurate with this approach, as identical devices are used to build all of the PMOS current sources. However, the layout requires a large area to accommodate not only the large number of devices but also the required minimum spacing between the devices. For instance, consider the MSB current source, which is built using 512 small PMOS devices arranged in a rectangular array of 8 rows and 64 devices in each row. Each PMOS device has dimensions of $1.2 \mu \mathrm{m} \times 5 \mu \mathrm{m}$. Along the length dimension, in addition to the device length of $5 \mu \mathrm{m}$, a spacing of $2 \mu \mathrm{m}$ is required for creating vias between adjacent devices, as illustrated in Figure 3.3. Here, in each row the devices are placed in such a way that the similar terminals of adjacent devices are overlapped on each other. For instance, in 
Figure 3.3, it can be seen that in each row the drain terminal of the first device is overlapped on the drain terminal of the second device. Similarly, the source terminal of the second device will be overlapped on the source terminal of the third device and so on. Therefore, the total length dimension of the MSB current source becomes

$$
L_{\text {Total }}=64 \times 5 \mu \mathrm{m}+65 \times 2 \mu \mathrm{m}=450 \mu \mathrm{m} .
$$

Along the width dimension, in addition to the device width of $1.2 \mu \mathrm{m}$, a spacing of 1.6 $\mu \mathrm{m}$ is left between two successive rows and the metal connections along the width dimension occupy an additional $5 \mu \mathrm{m}$ space, as illustrated in Figure 3.3. Therefore the total width dimension becomes

$$
W_{\text {Total }}=8 \times(5 \mu \mathrm{m}+1.2 \mu \mathrm{m})+7 \times 1.6 \mu \mathrm{m}=60.8 \mu \mathrm{m}
$$

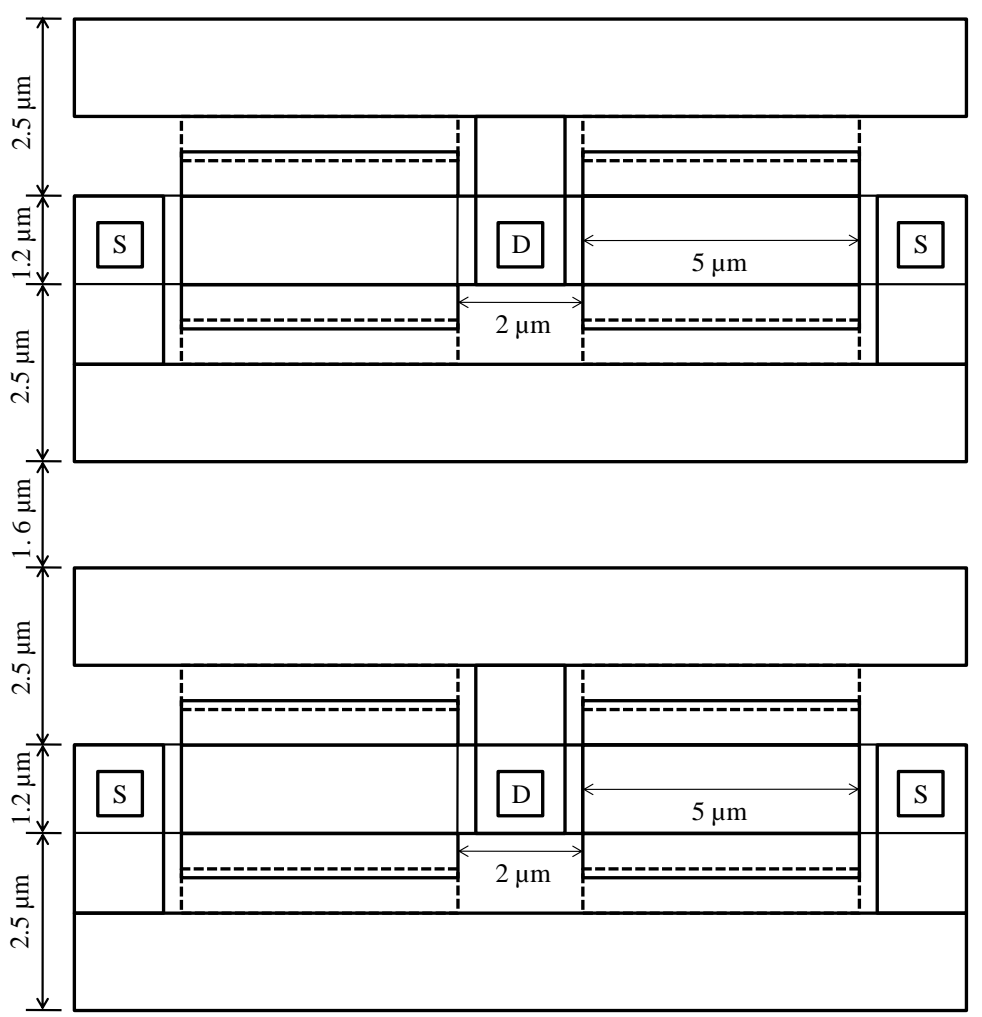

Figure 3.3 Block diagram showing the placement of the individual devices of the MSB current source, obtained using gate multiplication approach 
The foregoing calculations show that the MSB current source alone occupies an area of approximately $450 \mu \mathrm{m} \times 60.8 \mu \mathrm{m}$. Although this may be considered rather large, the design has the advantage that the bit current ratios are expected to be very close to the nominal values, even at high temperatures. As a result, this design is a good choice when chip area is not a primary concern.

\subsection{Design approach II: width customization}

As an alternative to gate multiplication, each of the binary-weighted PMOS current sources may be designed as a single device. The binary weightings of the bit currents are achieved by making the effective width of each device successively twice as large as that of the one before it, as we move from LSB to MSB. That is, the LSB current source is made of a single PMOS device of a given unit width, the next is made of a single PMOS device with twice the effective width, and so on. The MSB current source is made of a PMOS device whose effective width is $2^{N-1}$ times that of the LSB current source. For convenience in layout, the larger current sources may be divided into several separate devices. In this work, the four largest current sources are divided into separate devices.

The layout using width customization is considerably smaller than that using gate multiplication, because the spaces between the multiple devices that would make one current source are greatly reduced, or even eliminated. However, the design calculations are more complicated and the resulting bit currents are not as accurate. Given the desired effective widths of the PMOS current sources, the drawn widths have to be calculated from a relation such as

$$
W_{\text {eff }}=W_{\text {drawn }}-2 \Delta W,
$$


where $\Delta W$ is a parameter extracted from the detailed transistor models and depends on various factors such as mask fabrication techniques, photolithographic process and equipment, production quality control and source/drain junction depths [16].

To see how the drawn widths of the PMOS current sources are derived, consider the case where $m$ PMOS devices have to replace $n$ PMOS devices connected in parallel, each with width $W$. It is assumed that all of the original $n$ devices and the $m$ devices that replace them have the same length. Let $W_{\mathrm{d}}$ be the drawn width of each of the $n$ PMOS devices and $W_{\mathrm{d}}$ be the drawn width of each of the $m$ resultant PMOS devices. As the effective widths in these two cases have to be the same, we can obtain a relation between the two drawn widths as

$$
n \times\left(W_{\mathrm{d}}-2 \Delta W\right)=m \times\left(W_{\mathrm{d}}{ }^{\prime}-2 \Delta W\right) .
$$

Therefore, the drawn width of the resultant $m$-device current source is calculated from

$$
m \times W_{\mathrm{d}}{ }^{\prime}=n \times W_{\mathrm{d}}-2(n-m) \Delta W .
$$

For instance, consider a single PMOS device that has to replace two PMOS devices connected in parallel each with a drawn width $\left(W_{d}\right)$ of $1.2 \mu \mathrm{m}$. The drawn width of the single PMOS device can be obtained using Equation (3.1) with $n=2$ and $m=1$ as

$$
W_{\mathrm{d}}^{\prime}=2 \times 1.2-2 \times \Delta W \text {. }
$$

with $\Delta W$ obtained from the transistor models as $0.105 \mu \mathrm{m}$. The required drawn width of the single PMOS device is calculated as

$$
W_{\mathrm{d}}{ }^{\prime}=2.19 \mu \mathrm{m} \approx 2.2 \mu \mathrm{m} .
$$

The resolution of the transistor dimensions (both width and length) is limited to $0.1 \mu \mathrm{m}$ and hence the required drawn width is rounded-off to $2.2 \mu \mathrm{m}$, which will introduce a small error in the bit currents. 
Consider another case, where 4 PMOS devices connected in parallel have to replace 128 PMOS devices connected in parallel each with a drawn width $\left(W_{d}\right)$ of 1.2 $\mu \mathrm{m}$. The drawn width of each of these 4 PMOS devices can be obtained using Equation (3.1) as

$$
4 \times W_{\mathrm{d}}{ }^{\prime}=128 \times W_{\mathrm{d}}-2(128-4) \Delta W .
$$

Again using $\Delta W=0.105 \mu \mathrm{m}$ (obtained from the transistor models), the required drawn width of each of the 16 PMOS devices is calculated as

$$
W_{\mathrm{d}}{ }^{\prime}=31.89 \mu \mathrm{m} \approx 31.9 \mu \mathrm{m} .
$$

The drawn widths of all of the PMOS current sources are derived similarly. The LSB current source is obtained using a single PMOS device with drawn width of $W_{\mathrm{d}}{ }^{\prime}=$ $1.2 \mu \mathrm{m}$. The six smallest sources are constructed using a single MOSFET each, whereas the four largest are obtained by multiplying the devices by successive powers of two. The MSB current source is obtained by connecting 16 PMOS devices in parallel, each with calculated drawn width of $W_{\mathrm{d}}{ }^{\prime}=31.9 \mu \mathrm{m}$.

By simulating the current sources, it was found that there is a small error in the bit current produced by M15, which gets increased progressively as we move from M15 to M19. The error results from using an imprecise value of $\Delta W$ for the calculations, and from required values of $W_{\text {drawn }}$ that cannot be exactly resolved in the layout. In order to bring this error to a minimum level and to get the bit currents closer to the nominal values, the current sources M18 and M19 are re-designed to have drawn widths of $32 \mu \mathrm{m}$ and $32.3 \mu \mathrm{m}$ respectively, which are obtained by trial and error with a constraint of 0.1 $\mu \mathrm{m}$ resolution on the drawn widths. The drawn widths of all of the PMOS current sources thus obtained are summarized in Table 3.2. 
Table 3.2 Dimensions of the PMOS current sources obtained using width customization

\begin{tabular}{|c|c|c|c|}
\hline Current Source & $(W(\mu \mathrm{m}) \times L(\mu \mathrm{m}))$ & No. of Devices & Total Drawn Width $(\mu \mathrm{m})$ \\
\hline M10 (LSB) & $(1.2 \times 5)$ & 1 & 1.2 \\
\hline M11 & $(2.2 \times 5)$ & 1 & 4.2 \\
\hline M12 & $(4.2 \times 5)$ & 1 & 8.1 \\
\hline M13 & $(8.1 \times 5)$ & 1 & 16 \\
\hline M14 & $(16 \times 5)$ & 1 & 31.9 \\
\hline M15 & $(31.9 \times 5)$ & 1 & 63.8 \\
\hline M16 & $(31.9 \times 5)$ & 2 & 27.6 \\
\hline M17 & $(31.9 \times 5)$ & 4 & 516.8 \\
\hline M18 & $(32 \times 5)$ & 8 & \\
\hline M19 $($ MSB $)$ & $(32.3 \times 5)$ & 16 & \\
\hline
\end{tabular}

The resulting currents using the width customization approach will be imprecise first of all because the models themselves are approximate. Of course, the modeling errors will not be seen in the simulation results. However, the current errors are unavoidable even in the simulation because of the limited resolution of the transistor dimensions. Increasing the size of the LSB current source allows a corresponding increase in precision in the rest of the design. Hence, with this approach, there is a tradeoff between the area of the chip and the accuracy of the current ratios.

To give an idea of the difference in the overall device dimensions obtained using gate multiplication and width customization approaches; consider the MSB current source of a 10-bit DAC. Using gate multiplication this current source was laid out as 512 
small MOSFETS in a rectangular array with dimensions of $450 \mu \mathrm{m} \times 61 \mu \mathrm{m}$. Using width customization, the same current source was laid out as 16 larger MOSFETS in a rectangular array with dimensions of $111 \mu \mathrm{m} \times 37 \mu \mathrm{m}$. Including all of the current sources and their control circuitry, the total 10-bit DAC dimensions for the gate multiplication approach came out to be $495 \mu \mathrm{m} \times 135 \mu \mathrm{m}$, whereas the dimensions for the width customization approach are $150 \mu \mathrm{m} \times 92 \mu \mathrm{m}$. Figures 3.4 and 3.5 illustrate the floor plans of the gate-multiplied DAC and the width-customized DAC, respectively. The difference in the area occupied by the PMOS current sources of both the DAC designs can be observed from these figures.

A more detailed comparison of the two design approaches described in this chapter will be made in Chapter IV along with the test bench set up used for the simulations followed by the test results.

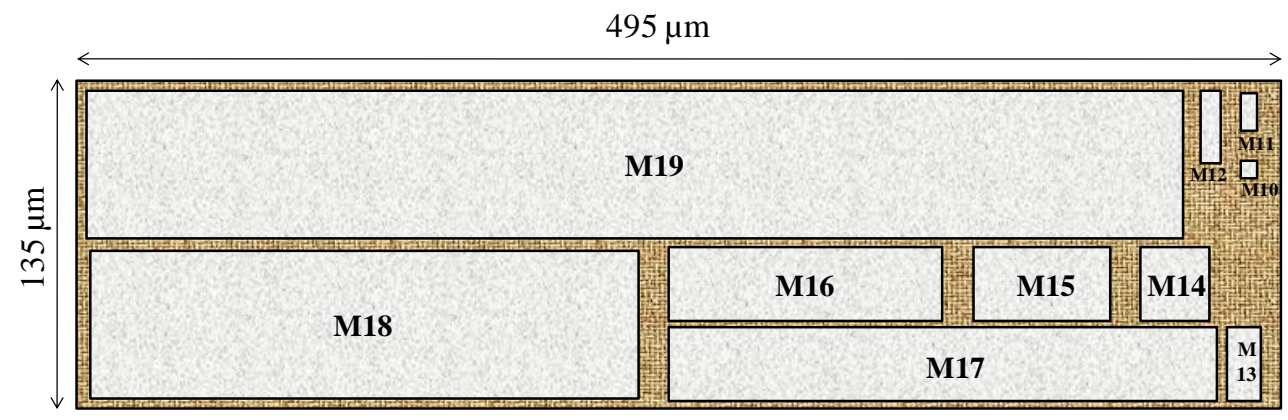

Figure 3.4 Floor plan showing the placement of current sources of 10-bit gate-multiplied DAC

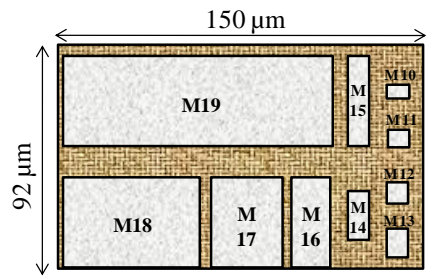

Figure 3.5 Floor plan showing the placement of current sources of 10-bit width-customized DAC 


\subsection{CMOS gate drive circuit}

The gate drive circuit shown in Figure 3.1 acts as an interface between the digital input and the PMOS current sources. Each current source is controlled by the digital input bit $\left(b_{i}\right)$ through the gate drive circuit, which is built from a PMOS-NMOS pair (M2i-M3i). The dimensions of the PMOS-NMOS pair are chosen to be the same for the six least significant bits and are different for the four most significant bits. The gate drive devices of the six least significant bits have dimensions of $(W \times L)=(8 \mu \mathrm{m} \times 0.5 \mu \mathrm{m})$ for the PMOS devices and $(W \times L)=(8 \mu \mathrm{m} \times 0.8 \mu \mathrm{m})$ for the NMOS devices. The gate drive devices for the four most significant bits have the same length but the widths of the PMOS and NMOS devices are scaled by higher powers of two. This is because, as we move towards the most significant bits, the gate areas of the PMOS current sources get bigger, and so the gate driver devices must increase in size to maintain an acceptable speed of operation. The maximum rise and fall times of the digital input being applied to the gate of the PMOS current sources through the gate drive circuit have been found from simulations to be $198.8 \mathrm{~ns}$ and $233.7 \mathrm{~ns}$ respectively. Up to the precision allowed by the simulations, these rise and fall times also apply to the resulting bit currents.

\subsection{Choice of bias voltage and temperature insensitive operation}

The choice of $V_{\text {BIAS }}$ plays a vital role in the implementation of the proposed DAC, whose one-bit implementation is shown in Figure 3.1. If $V_{\mathrm{BIAS}}$ is chosen to be equal to 0 $\mathrm{V}\left(\mathrm{V}_{G S}=\mathrm{V}_{D D}=3 \mathrm{~V}\right)$, then the PMOS current sources (when active or turned $\mathrm{ON}$ ) are in the triode region. Hence any small change in the drain-to-source voltage, whether caused by variations in the summing node voltage or the power supply voltage, can cause a significant change in the current delivered by these sources; this situation is illustrated by 
Curve [a] of Figure 3.6. On the other hand if $V_{\mathrm{BIAS}}$ is chosen to be greater than $0 \mathrm{~V}$ such that the current sources are deep in the saturation region, then small changes in the drainto-source voltage will not cause significant variations in the current; this situation is illustrated by Curve [b] of Figure 3.6, which corresponds to $\mathrm{V}_{B I A S}=1 \mathrm{~V}\left(\mathrm{~V}_{G S}=2 \mathrm{~V}\right)$.

In addition to helping the DAC to operate independent of voltage variations, the choice of $V_{\text {BIAS }}$ may be made to help the DAC to maintain constant currents despite variations in temperature [17]. To maintain constant-current operation over a wide temperature range the PMOS current sources are operated with the gates at the Zero Temperature Coefficient (ZTC) voltage [4], [5].

The ZTC voltage is found by plotting the transconductance characteristics of a PMOS device at several different temperatures, and finding a point where all of the curves intersect. Figure 3.7 shows typical device characteristics at $27^{\circ} \mathrm{C}$ and $125^{\circ} \mathrm{C}$. The curves corresponding to any two temperatures intersect at approximately the same point. For this device, $V_{\mathrm{BIAS}}$ is chosen as $933.2 \mathrm{mV}$, which is the ZTC voltage of the PMOS device. The ZTC voltage changes by few millivolts when the width of a MOSFET is changed. The bias voltage $\left(V_{\mathrm{BIAS}}\right)$ is simulated as an ideal voltage source and also using a bias generation circuit while evaluating the performance of the proposed designs from simulations.

The simulation setup and test results used to evaluate the performance of the proposed 10-bit DAC designs at different temperatures are discussed in Chapter IV.

\subsection{Bias generation circuit}

The bias voltage $\left(V_{\mathrm{BIAS}}\right)$ required for the PMOS current sources can be obtained using a bias generation circuit shown in Figure 3.8. It is built using a single PMOS and 
two NMOS transistors in series, each in a diode-connected configuration. The transistor dimensions are $(W \times L)=(2.4 \mu \mathrm{m} \times 5 \mu \mathrm{m})$ for the PMOS device $(\mathrm{P} 1)$ and $(W \times L)=(1.2$ $\mu \mathrm{m} \times 5 \mu \mathrm{m})$ for each of the NMOS devices (N1, N2). The current through the circuit is approximately $1.052 \mu \mathrm{A}$ at $27^{\circ} \mathrm{C}$.

Figure 3.9 shows the variation in the output voltage $\left(V_{\mathrm{BIAS}}\right)$ of the bias generation circuit when the temperature is varied between $27^{\circ} \mathrm{C}$ and $125^{\circ} \mathrm{C}$. It is observed that the output voltage matches the desired bias voltage at $27^{\circ} \mathrm{C}$ and varies only by approximately $0.9 \mathrm{mV}$ over the entire temperature range. When the circuit is re-designed to match the desired bias voltage in the middle of the temperature range, the variation in the output voltage over the entire temperature range turns out to be greater. Hence, the bias generation circuit tuned to $27^{\circ} \mathrm{C}$ is used for creating $V_{\mathrm{BIAS}}$ in this work; this is expected to keep the currents delivered by the PMOS sources close to the nominal values even at high temperatures.

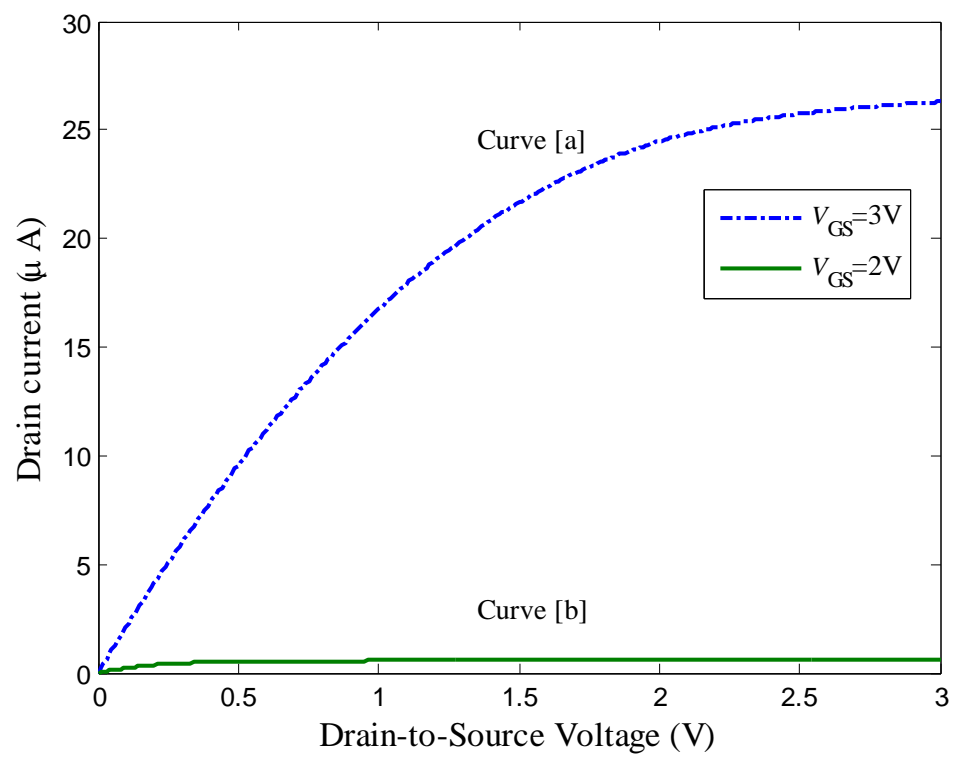

Figure 3.6 Plot showing the I-V characteristics of a PMOS transistor $(W=1.2 \mu \mathrm{m}$; $L=5 \mu \mathrm{m}$ ) for different values of $V_{\mathrm{BIAS}}$ 


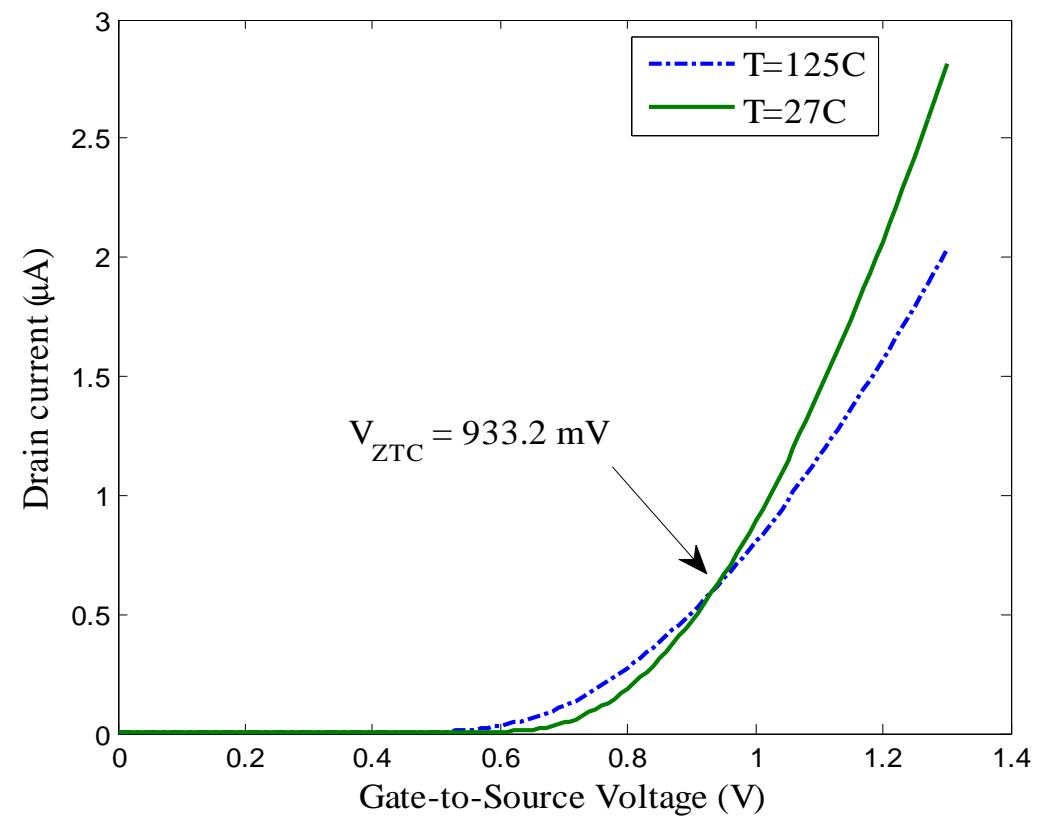

Figure 3.7 Simulated transconductance characteristics of a PMOS transistor $(W=1.2 \mu \mathrm{m} ; L=5 \mu \mathrm{m})$ at two different temperatures $\left(27^{\circ} \mathrm{C}\right.$ and $\left.125^{\circ} \mathrm{C}\right)$

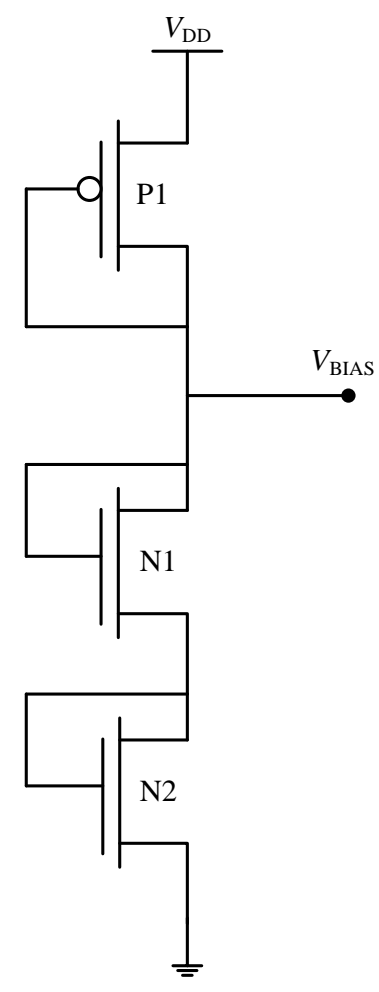

Figure 3.8 Bias generation circuit 


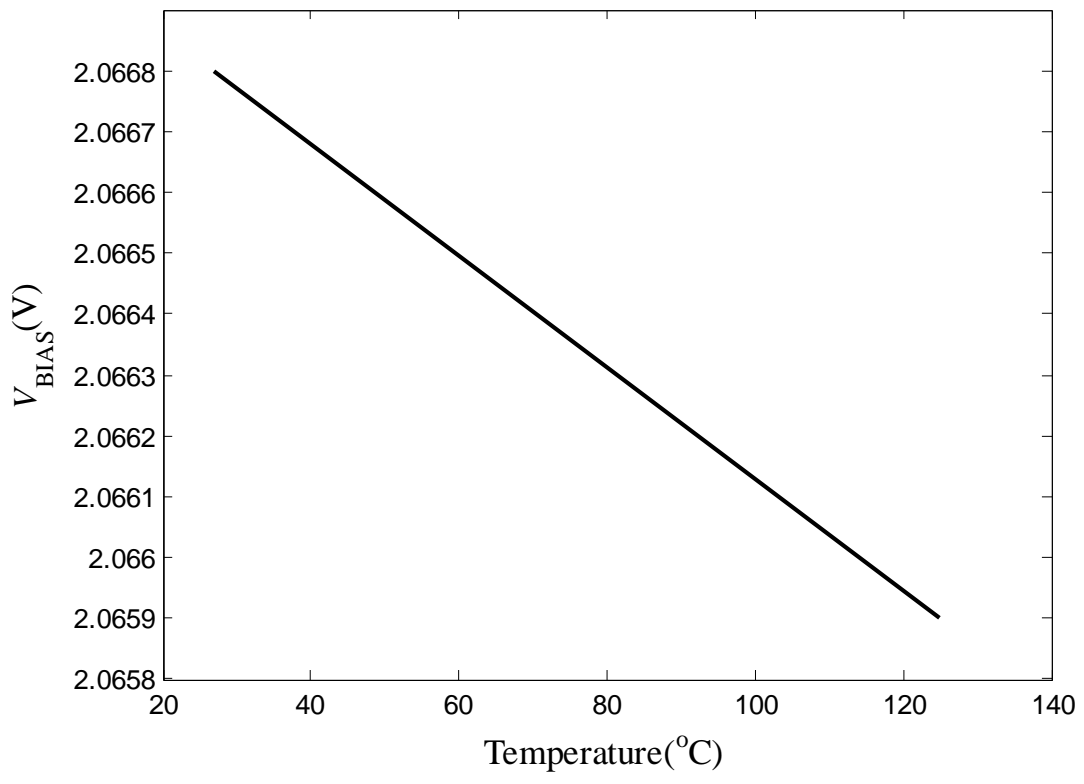

Figure 3.9 Plot showing the variation in the bias voltage $\left(V_{\mathrm{BIAS}}\right)$ with temperature 


\section{CHAPTER IV \\ SIMULATION SETUP AND RESULTS}

The proposed 10-bit DAC designs obtained from two different approaches (gate multiplication and width customization) have been laid out for a $0.5-\mu \mathrm{m}$ SOI process using a 3-V unipolar supply. The approximate die dimensions were $495 \mu \mathrm{m} \times 135 \mu \mathrm{m}$ for the gate-multiplied DAC and $150 \mu \mathrm{m} \times 92 \mu \mathrm{m}$ for the width-customized DAC. The transistor-level implementation and simulation of the proposed DAC designs have been accomplished by the use of Cadence ${ }^{\circledR}$ ICFB design environment. The transistor-level schematics have been implemented using the Schematic Editor and the simulations have been carried out using the Virtuoso ${ }^{\circledR}$ Spectre® Circuit Simulator. In the following sections, the simulation results are presented and discussed, and various performance measures of the two DAC designs are evaluated.

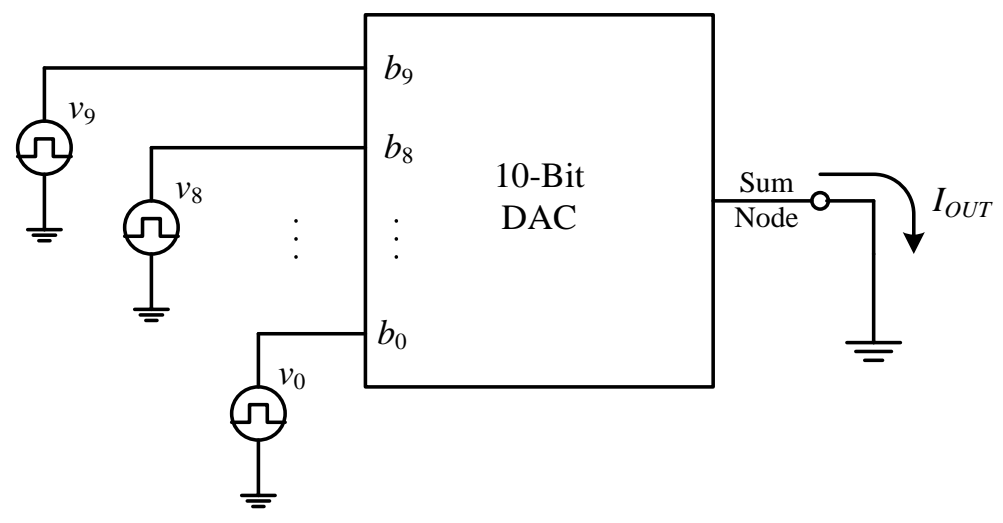

Figure 4.1 Test setup for the 10-bit DAC simulation 
Figure 4.1 shows the test setup used for simulating the proposed 10-bit DAC designs. The digital inputs $\left(b_{9}, b_{8}, \ldots, b_{0}\right)$ to the CMOS drivers are simulated as ideal voltage sources $\left(v_{9}, v_{8}, \ldots, v_{0}\right)$ and the output current $\left(I_{\text {OUT }}\right)$ is obtained from the sum node to which all ten of the current sources are connected. The bias voltage $\left(V_{\mathrm{BIAS}}\right)$ required for the current sources is simulated first as an ideal voltage source and then using the bias generation circuit discussed in Section 3.6. The same setup is used for simulating both of the 10-bit DAC designs, except that the designs of the PMOS current sources are changed accordingly.

Figure 4.2 shows the simulation results of the individual source currents that result from the gate-multiplied design of the 10-bit DAC. The current sources are turned ON sequentially, one at a time, starting with the MSB. The results are shown for two different temperatures, $27^{\circ} \mathrm{C}$ and $125^{\circ} \mathrm{C}$. The bias voltage ( $V_{\mathrm{BIAS}}$ ) for the gate drivers is supplied by an ideal source. Figure 4.3 shows the same simulation results for the widthcustomized design.

From Figure 4.2 and Figure 4.3, it can be seen that the gate-multiplied design exhibits better performance than the width-customized design, not only in terms of the ratios of the bit currents, but also in terms of the absolute currents delivered by the PMOS current sources over temperature, because the current sources of the gate-multiplied design are built using identical PMOS devices and hence the ZTC voltage remains the same for all the PMOS current sources. By contrast, the current sources of the widthcustomized DAC are not identical and hence the ZTC voltages differ by a few millivolts from one device to another. Thus, it is not possible to choose one fixed $V_{\text {BIAS }}$ that puts all 
the current sources at their ZTC points, and the result is small variations in the bit currents over temperatures.

The same set of simulations is performed by replacing the ideal bias voltage source with the bias generation circuit and the results obtained are shown in Figure 4.4 and Figure 4.5. It can be observed that the output current is still reasonably close to the nominal value but the errors in the bit currents in this case are slightly higher than the errors in the case of the ideal bias voltage source.

The LSB and MSB currents are shown in Table 4.1 as representative values of the currents shown in Figures 4.2 - 4.5. It can be seen that the PMOS current sources of the width-customized DAC design deliver currents with a maximum error of about $5.6 \%$ using the ideal bias voltage source and $6.1 \%$ using the bias generation circuit. The current sources of the gate-multiplied DAC design are somewhat more stable over temperature with a maximum error of about $0.4 \%$ using the ideal bias voltage source and $0.48 \%$ using the bias generation circuit.

The ratios between consecutive bit currents (whose ideal value is two) were found at two different temperatures $\left(27^{\circ} \mathrm{C}\right.$ and $\left.125^{\circ} \mathrm{C}\right)$. The minimum and maximum ratios between any two consecutive bit currents are shown in Table 4.2. It can be seen that the width-customized DAC design keep the bit current ratios close to two with a maximum error of about $3.8 \%$ using the ideal bias voltage source and $4.1 \%$ using the bias generation circuit. The gate-multiplied design keeps more consistent current ratios at any given temperature with a maximum error of about $0.2 \%$ using the ideal bias voltage source and $0.25 \%$ using the bias generation circuit. 
The transfer characteristic of the gate-multiplied DAC design for all combinations of the digital input, obtained from simulations, is shown in Figure 4.6. The results are shown for two different temperatures, $27^{\circ} \mathrm{C}$ and $125^{\circ} \mathrm{C}$, along with the ideal transfer characteristic. The bias voltage ( $\left.V_{\mathrm{BIAS}}\right)$ for the gate drivers is supplied by an ideal source. Figure 4.7 shows the same results for the width-customized design. From the expanded versions of Figure 4.6 and Figure 4.7, which are obtained from the worst parts of the transfer characteristics, it can be seen that the error in the current levels of the gatemultiplied DAC is smaller than that in the width-customized DAC.

A similar set of simulations is carried out using the bias generation circuit and the results obtained are shown in Figure 4.8 and Figure 4.9. It can be seen that the deviation of the output current from the ideal value is slightly higher in this case when compared to the results obtained using the ideal bias voltage source.

Table 4.1 Currents delivered by the PMOS current sources

\begin{tabular}{|c|c|c|c|c|c|c|c|c|c|c|}
\hline & \multicolumn{4}{|c|}{} & \multicolumn{3}{|c|}{ Ideal voltage source } & \multicolumn{3}{c|}{ Bias generation circuit } \\
\cline { 2 - 11 } & \multicolumn{2}{|c|}{ Nominal } & \multicolumn{2}{|c|}{$27^{\circ} \mathrm{C}$} & \multicolumn{2}{c|}{$125^{\circ} \mathrm{C}$} & \multicolumn{2}{|c|}{$27^{\circ} \mathrm{C}$} & \multicolumn{2}{c|}{$125^{\circ} \mathrm{C}$} \\
\cline { 2 - 11 } & $\begin{array}{c}\text { LSB } \\
(\mu \mathrm{A})\end{array}$ & $\begin{array}{c}\text { MSB } \\
(\mu \mathrm{A})\end{array}$ & $\begin{array}{c}\text { LSB } \\
(\mu \mathrm{A})\end{array}$ & $\begin{array}{c}\text { MSB } \\
(\mu \mathrm{A})\end{array}$ & $\begin{array}{c}\text { LSB } \\
(\mu \mathrm{A})\end{array}$ & $\begin{array}{c}\text { MSB } \\
(\mu \mathrm{A})\end{array}$ & $\begin{array}{c}\text { LSB } \\
(\mu \mathrm{A})\end{array}$ & $\begin{array}{c}\text { MSB } \\
(\mu \mathrm{A})\end{array}$ & $\begin{array}{c}\text { LSB } \\
(\mu \mathrm{A})\end{array}$ & $\begin{array}{c}\text { MSB } \\
(\mu \mathrm{A})\end{array}$ \\
\hline $\begin{array}{c}\text { Gate-multiplied } \\
\text { DAC }\end{array}$ & 0.596 & 305.1 & 0.596 & 305.0 & 0.597 & 306.4 & 0.596 & 305.0 & 0.598 & 306.6 \\
\hline $\begin{array}{c}\text { Width- } \\
\text { customized DAC }\end{array}$ & 0.596 & 305.1 & 0.596 & 303.4 & 0.597 & 288.9 & 0.596 & 303.4 & 0.598 & 287.5 \\
\hline
\end{tabular}

Table 4.2 Ratios between consecutive bit currents

\begin{tabular}{|c|c|c|c|c|c|c|c|c|}
\hline \multirow{2}{*}{} & \multicolumn{3}{|c|}{ Ideal voltage source } & \multicolumn{3}{c|}{ Bias generation circuit } \\
\cline { 2 - 9 } & \multicolumn{2}{|c|}{$27^{\circ} \mathrm{C}$} & \multicolumn{2}{c|}{$125^{\circ} \mathrm{C}$} & \multicolumn{2}{c|}{$27^{\circ} \mathrm{C}$} & \multicolumn{2}{c|}{$125^{\circ} \mathrm{C}$} \\
\cline { 2 - 9 } & Min & Max & Min & Max & Min & Max & Min & Max \\
\hline Gate-multiplied DAC & 1.998 & 2.004 & 2.000 & 2.003 & 1.998 & 2.004 & 1.999 & 2.005 \\
\hline Width-customized DAC & 1.975 & 2.018 & 1.926 & 2.019 & 1.975 & 2.018 & 1.921 & 2.024 \\
\hline
\end{tabular}


Figure 4.10 shows the simulated INL characteristics of the 10-bit gate-multiplied DAC using an ideal source for $V_{\mathrm{BIAS}}$ for all the 1024 digital input combinations. The results are shown for two different temperatures, $27^{\circ} \mathrm{C}$ and $125^{\circ} \mathrm{C}$. Figure 4.11 shows the same simulation results for the width-customized design. From Figure 4.10 and Figure 4.11, it can be seen that INL error of the gate-multiplied design is slightly smaller than that of the width customized design, at both temperatures.

The simulated INL characteristics that are obtained using the bias generation circuit for $V_{\text {BIAS }}$ are shown in Figure 4.12 and Figure 4.13. It can be seen that the INL error in this case is slightly higher than the error in the case of the ideal bias voltage source.

Figure 4.14 shows the simulated DNL characteristics of the 10-bit gate-multiplied DAC design using an ideal source for $V_{\text {BIAS }}$ for all the 1024 digital input combinations. The results are shown for two different temperatures, $27^{\circ} \mathrm{C}$ and $125^{\circ} \mathrm{C}$. Figure 4.15 shows the same simulation results for width-customized design. From Figure 4.14 and Figure 4.15, it can be seen that DNL error for the gate-multiplied design is slightly smaller than that for the width-customized design, at both temperatures.

Table 4.3 Maximum Values of DNL \& INL

\begin{tabular}{|c|c|c|c|c|c|c|c|c|}
\hline \multirow{2}{*}{} & \multicolumn{3}{|c|}{ Ideal voltage source } & \multicolumn{3}{c|}{ Bias generation circuit } \\
\cline { 2 - 9 } & \multicolumn{2}{|c|}{$27^{\circ} \mathrm{C}$} & \multicolumn{2}{c|}{$125^{\circ} \mathrm{C}$} & \multicolumn{2}{c|}{$27^{\circ} \mathrm{C}$} & \multicolumn{2}{c|}{$125^{\circ} \mathrm{C}$} \\
\cline { 2 - 9 } & $\begin{array}{c}\text { DNL } \\
\text { (LSB) }\end{array}$ & $\begin{array}{c}\text { INL } \\
\text { (LSB) }\end{array}$ & $\begin{array}{c}\text { DNL } \\
\text { (LSB) }\end{array}$ & $\begin{array}{c}\text { INL } \\
(\text { LSB })\end{array}$ & $\begin{array}{c}\text { DNL } \\
\text { (LSB })\end{array}$ & $\begin{array}{c}\text { INL } \\
\text { (LSB) }\end{array}$ & $\begin{array}{c}\text { DNL } \\
\text { (LSB) }\end{array}$ & $\begin{array}{c}\text { INL } \\
\text { (LSB) }\end{array}$ \\
\hline Gate-multiplied DAC & 0.568 & 0.854 & 0.606 & 0.976 & 0.568 & 0.854 & 0.612 & 0.993 \\
\hline Width-customized DAC & 0.587 & 0.932 & 0.632 & 1.075 & 0.587 & 0.932 & 0.641 & 1.108 \\
\hline
\end{tabular}


The simulated DNL characteristics that are obtained using the bias generation circuit for $V_{\text {BIAS }}$ are shown in Figure 4.16 and Figure 4.17. It can be seen that the DNL error in this case is slightly higher than the error in the case of the ideal bias voltage source.

The maximum values of differential non-linearity (DNL) and integral nonlinearity (INL) associated with the proposed designs have been calculated from the simulation results. The calculated values are shown in Table 4.3, from which it can be observed that the non-linearity associated with the width-customized DAC design is slightly higher than the gate-multiplied DAC design at both temperatures. 


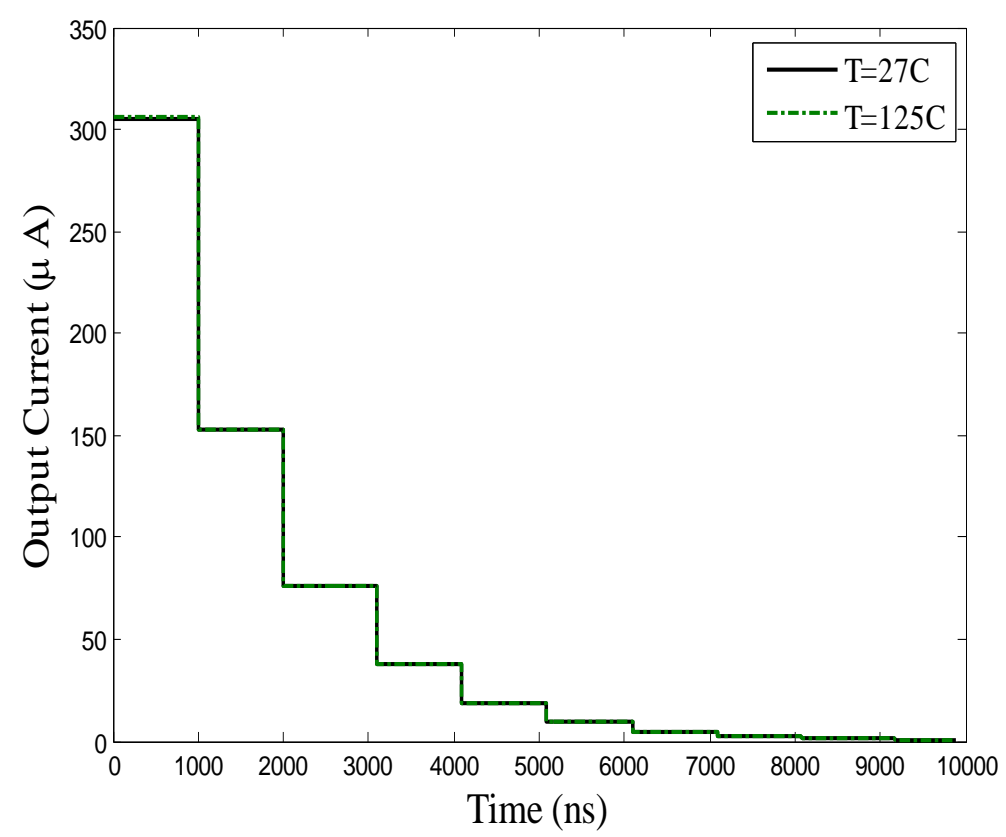

Figure 4.2 Output of 10-bit gate-multiplied DAC at $27^{\circ} \mathrm{C}$ and $125^{\circ} \mathrm{C}$ obtained using ideal bias voltage source

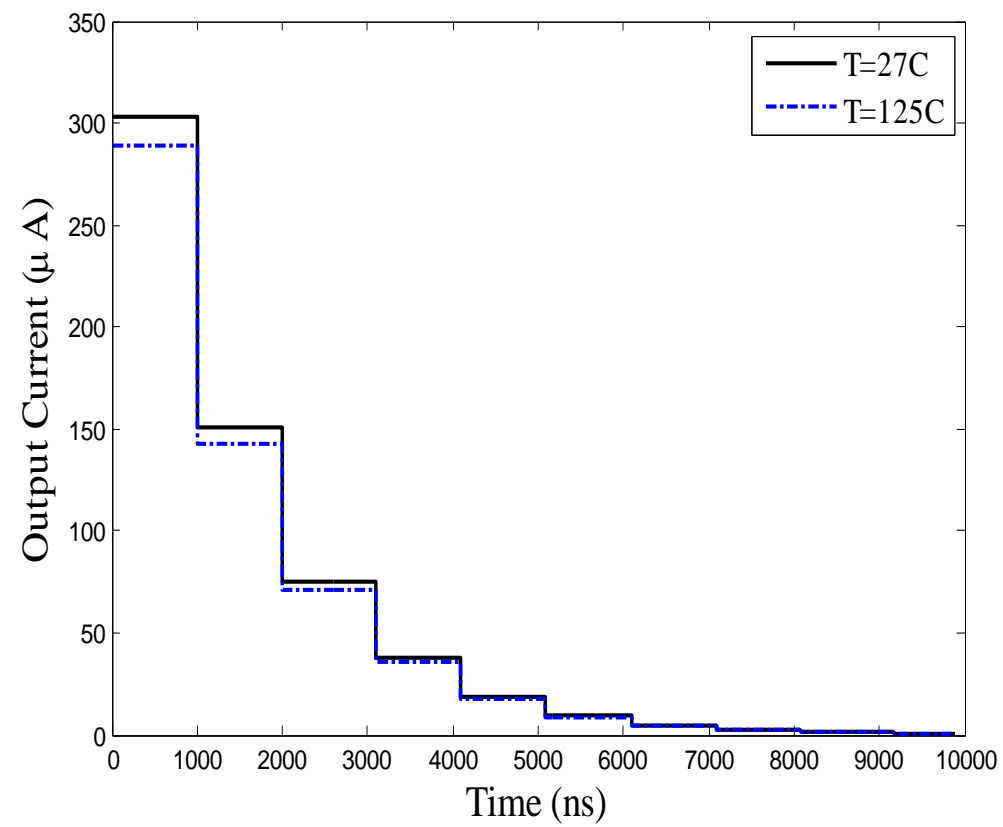

Figure 4.3 Output of 10-bit width-customized DAC at $27^{\circ} \mathrm{C}$ and $125^{\circ} \mathrm{C}$ obtained using ideal bias voltage source 


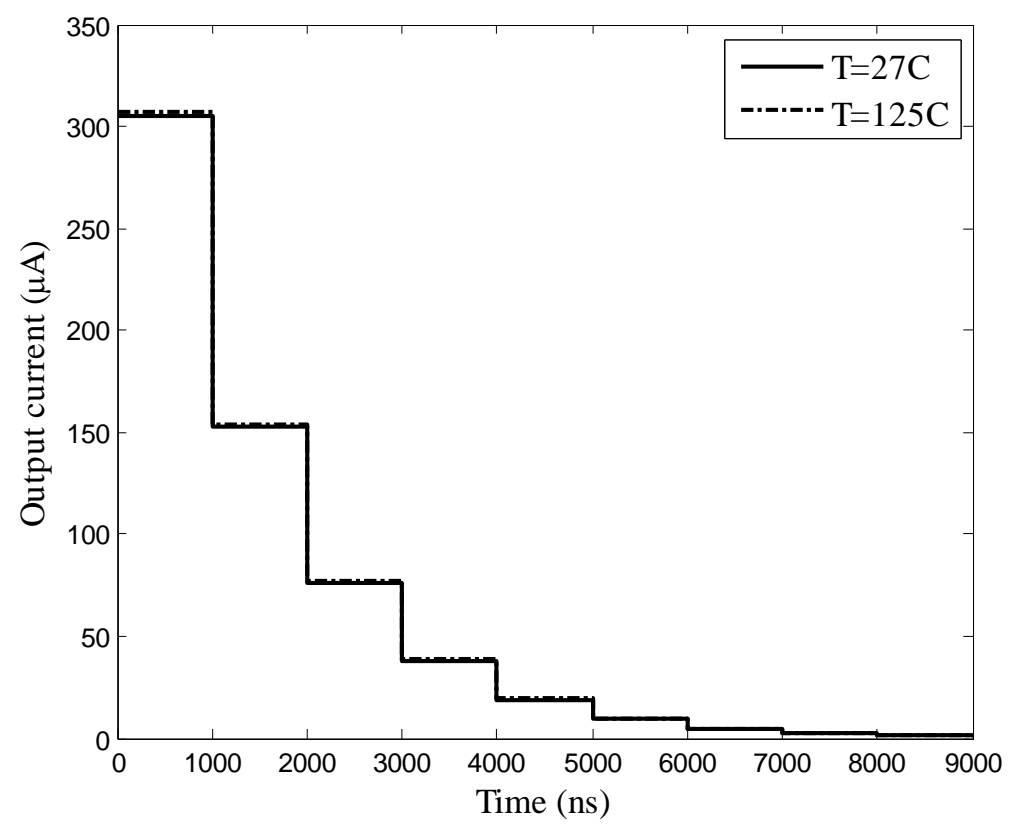

Figure 4.4 Output of the 10-bit gate-multiplied DAC at $27^{\circ} \mathrm{C}$ and $125^{\circ} \mathrm{C}$ obtained using bias generation circuit

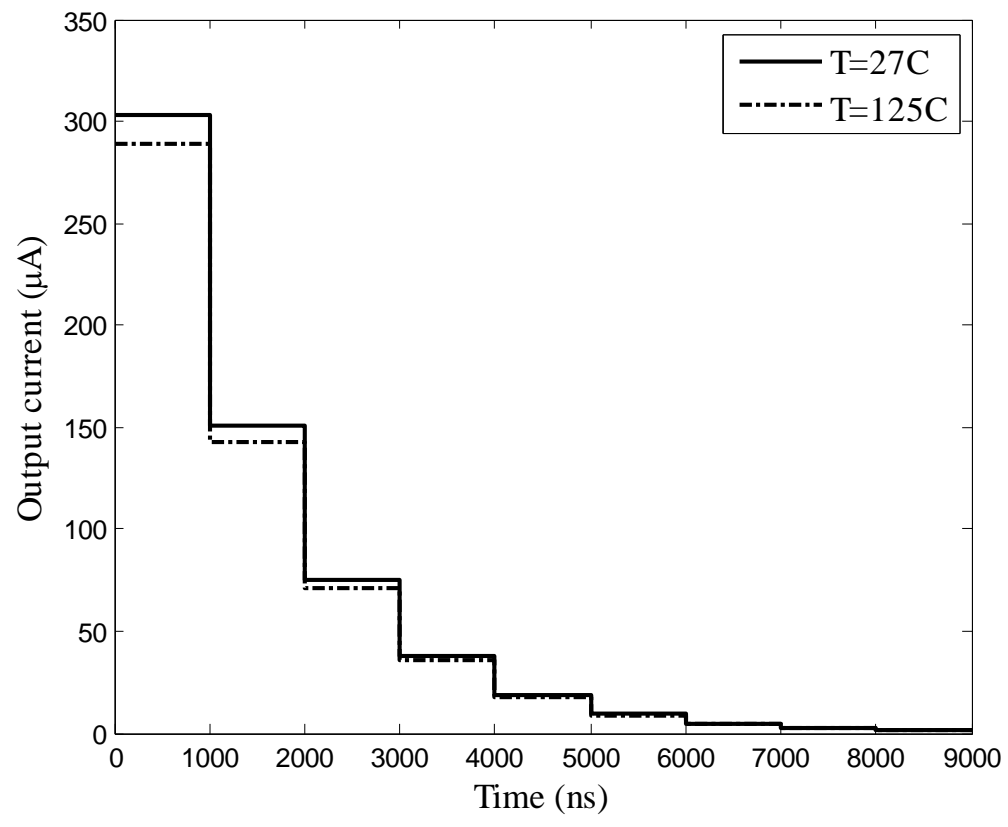

Figure 4.5 Output of the 10-bit width-customized DAC at $27^{\circ} \mathrm{C}$ and $125^{\circ} \mathrm{C}$ obtained using bias generation circuit 


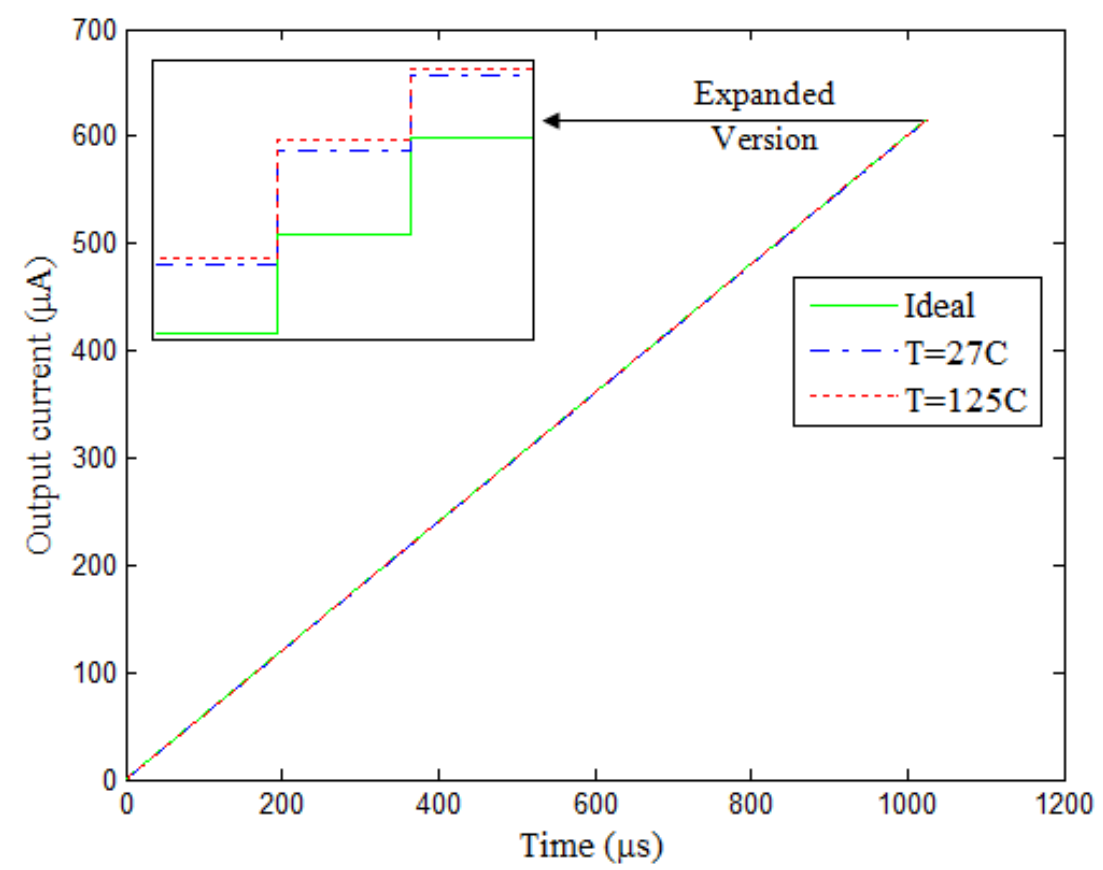

Figure 4.6 Transfer characteristics of 10-bit gate-multiplied DAC at $27^{\circ} \mathrm{C}$ and $125^{\circ} \mathrm{C}$ obtained using ideal bias voltage source, along with the nominal transfer characteristic

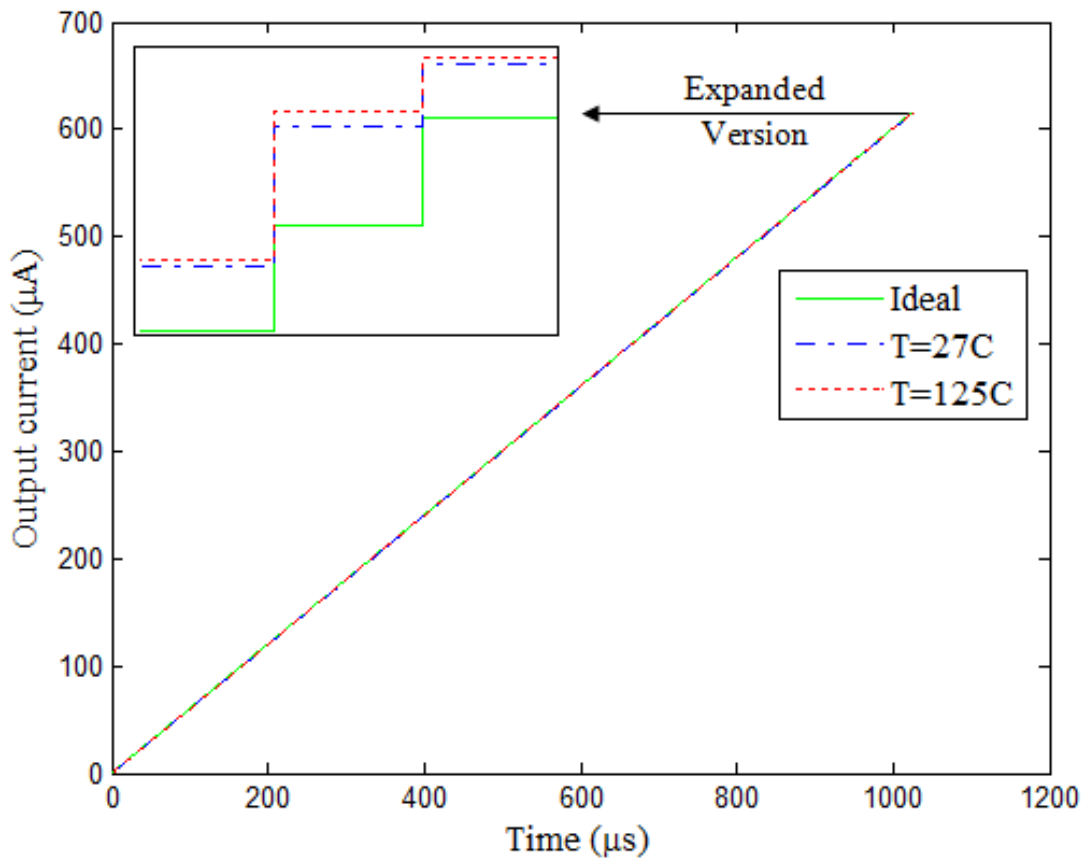

Figure 4.7 Transfer characteristics of 10-bit width-customized DAC at $27^{\circ} \mathrm{C}$ and $125^{\circ} \mathrm{C}$ obtained using ideal bias voltage source, along with the nominal transfer characteristic 


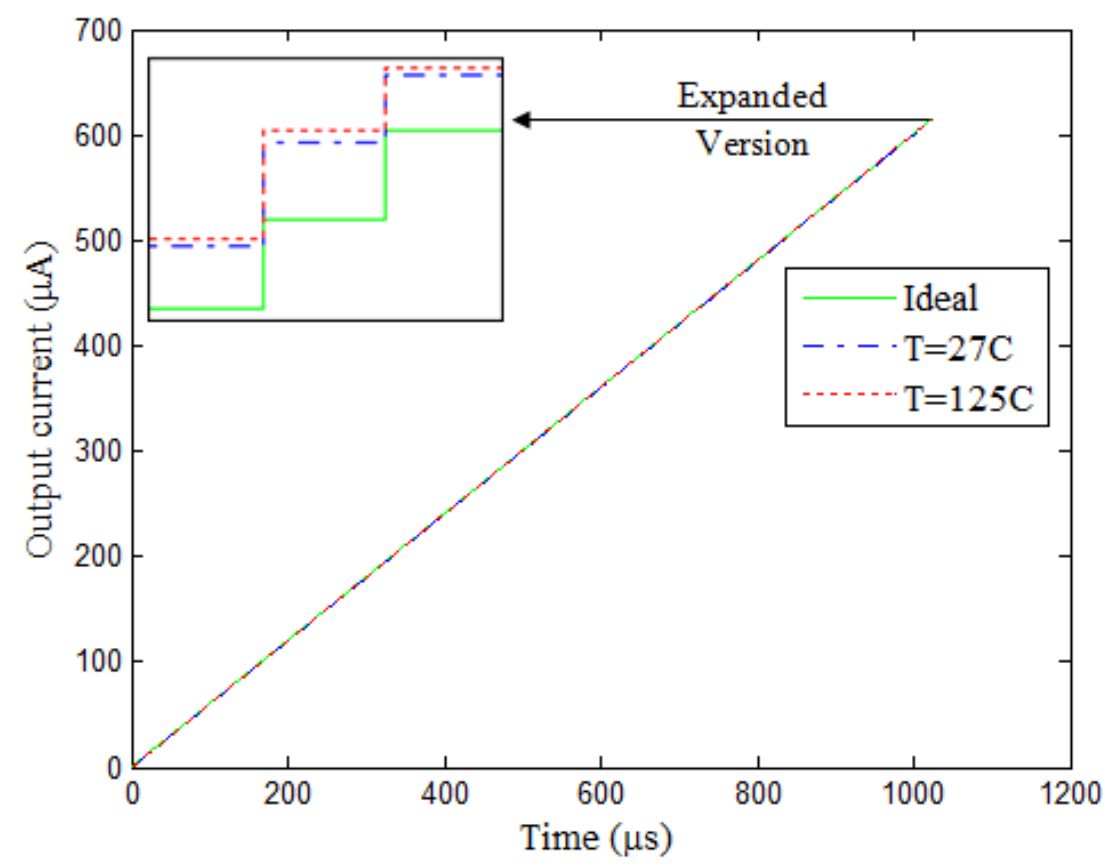

Figure 4.8 Transfer characteristics of 10-bit gate-multiplied DAC at $27^{\circ} \mathrm{C}$ and $125^{\circ} \mathrm{C}$ obtained using bias generation circuit, along with the nominal transfer characteristic

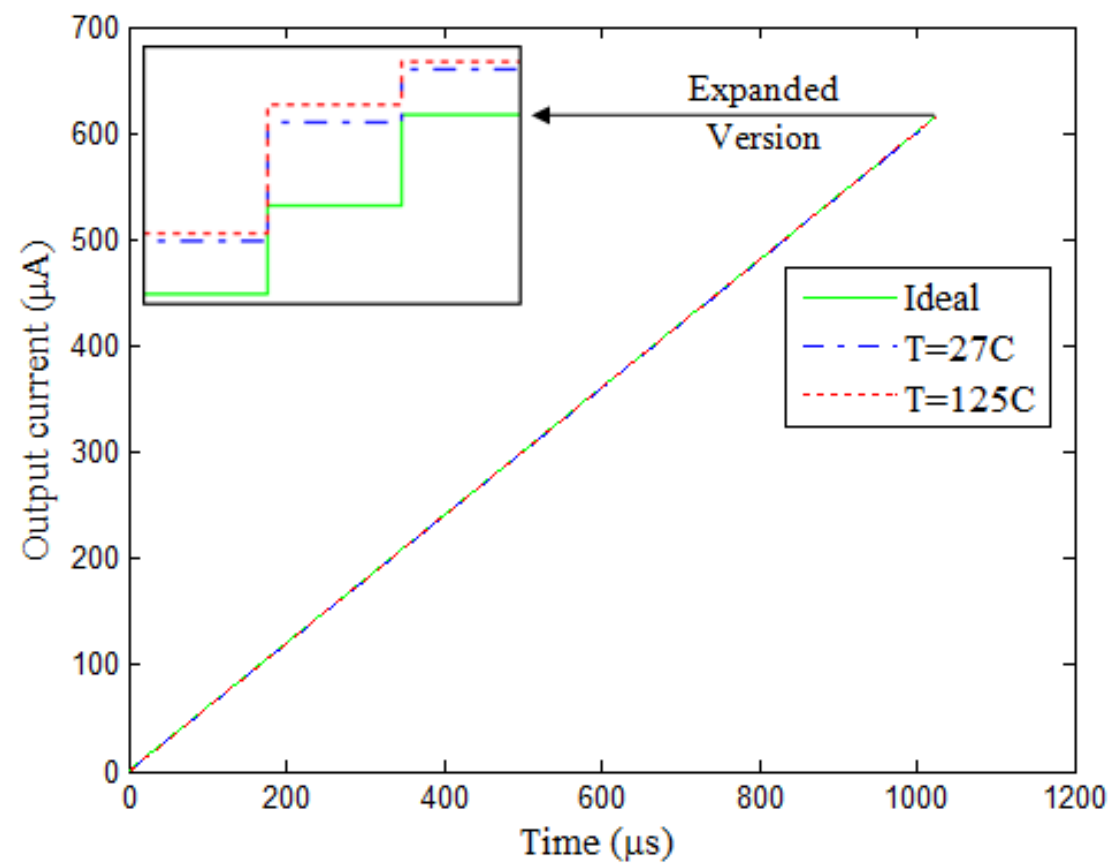

Figure 4.9 Transfer characteristics of 10-bit width-customized DAC at $27^{\circ} \mathrm{C}$ and $125^{\circ} \mathrm{C}$ obtained using bias generation circuit, along with the nominal transfer characteristic 


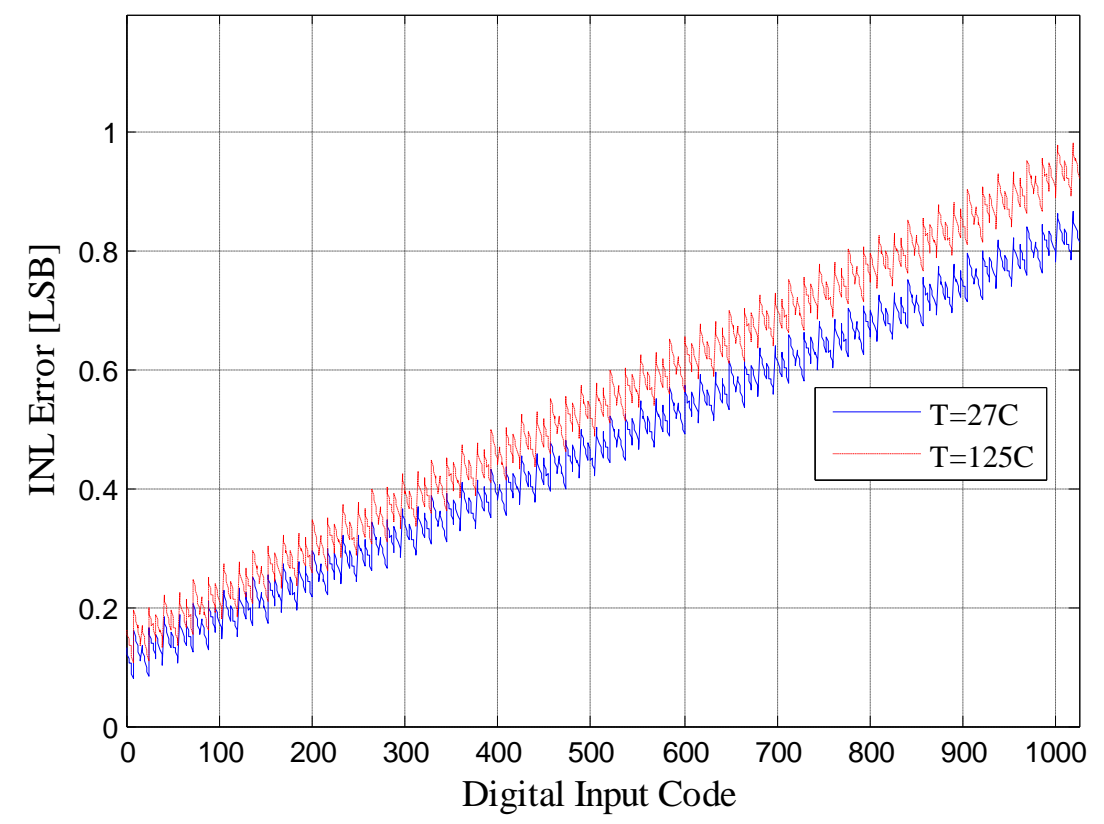

Figure 4.10 Simulated INL characteristics of 10-bit gate-multiplied DAC using ideal bias voltage source at $27^{\circ} \mathrm{C}$ and $125^{\circ} \mathrm{C}$

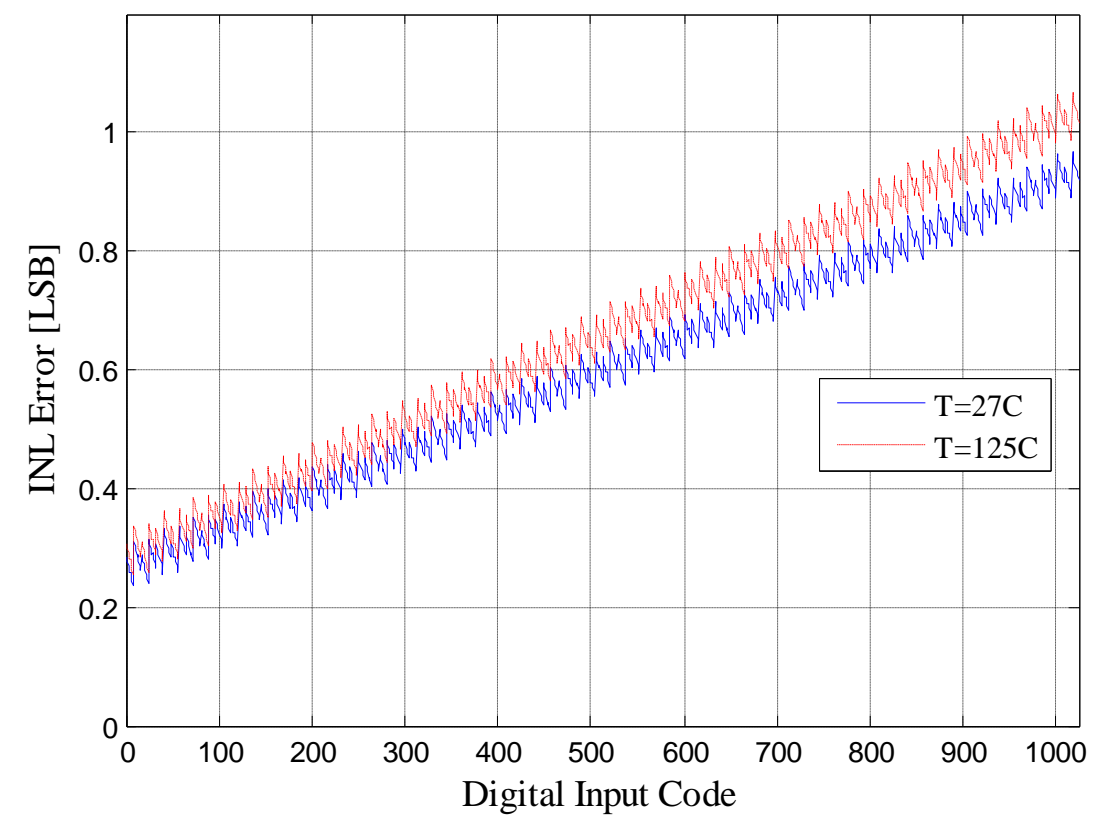

Figure 4.11 Simulated INL characteristics of 10-bit width-customized DAC using ideal bias voltage source at $27^{\circ} \mathrm{C}$ and $125^{\circ} \mathrm{C}$ 


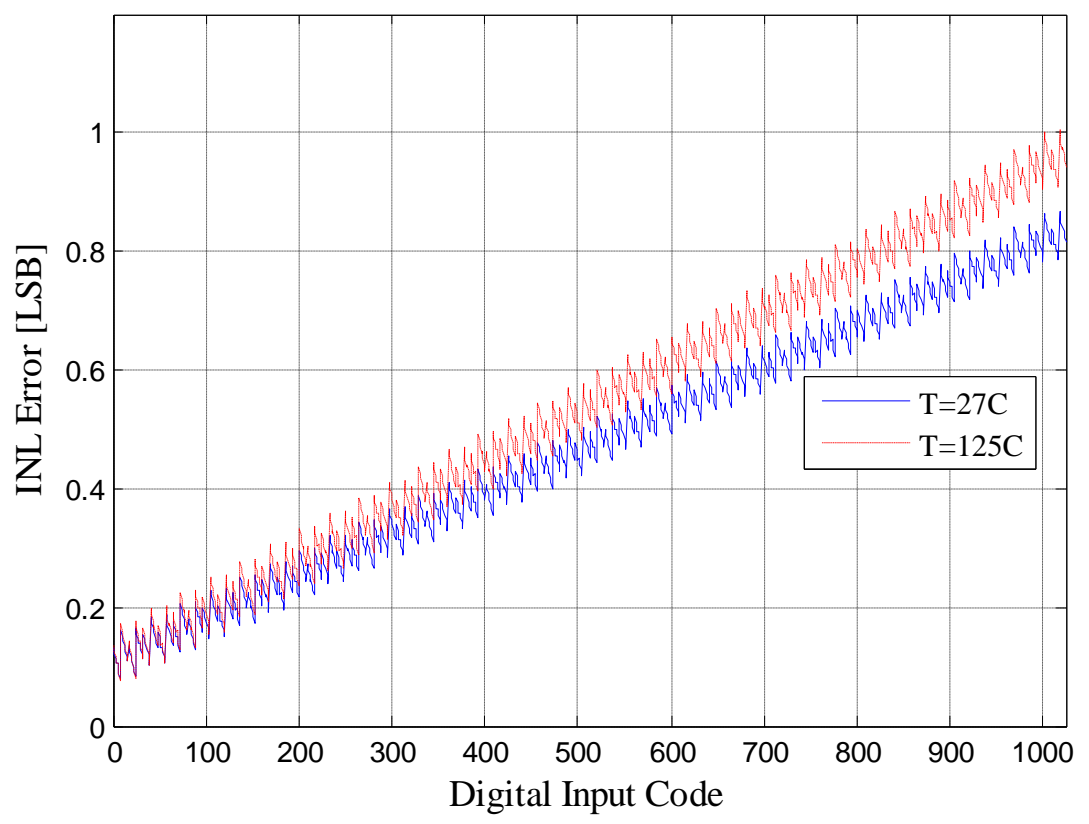

Figure 4.12 Simulated INL characteristics of 10-bit gate-multiplied DAC using bias generation circuit at $27^{\circ} \mathrm{C}$ and $125^{\circ} \mathrm{C}$

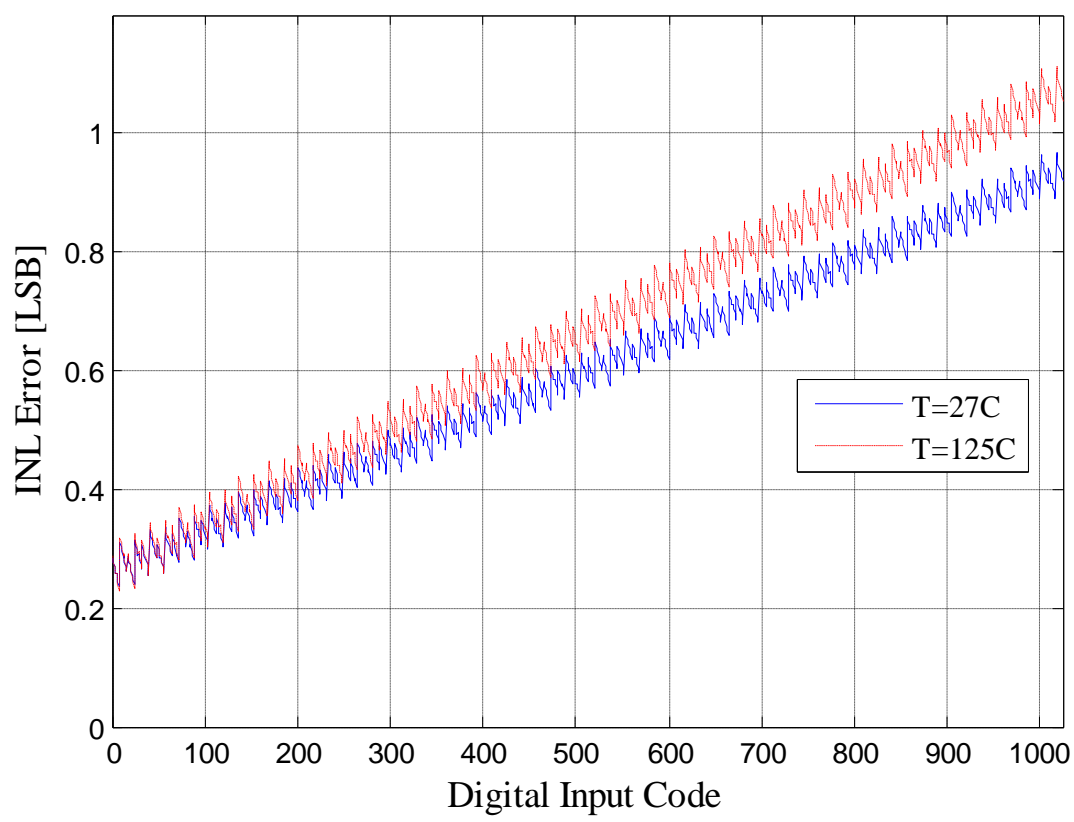

Figure 4.13 Simulated INL characteristics of 10-bit width-customized DAC using bias generation circuit at $27^{\circ} \mathrm{C}$ and $125^{\circ} \mathrm{C}$ 


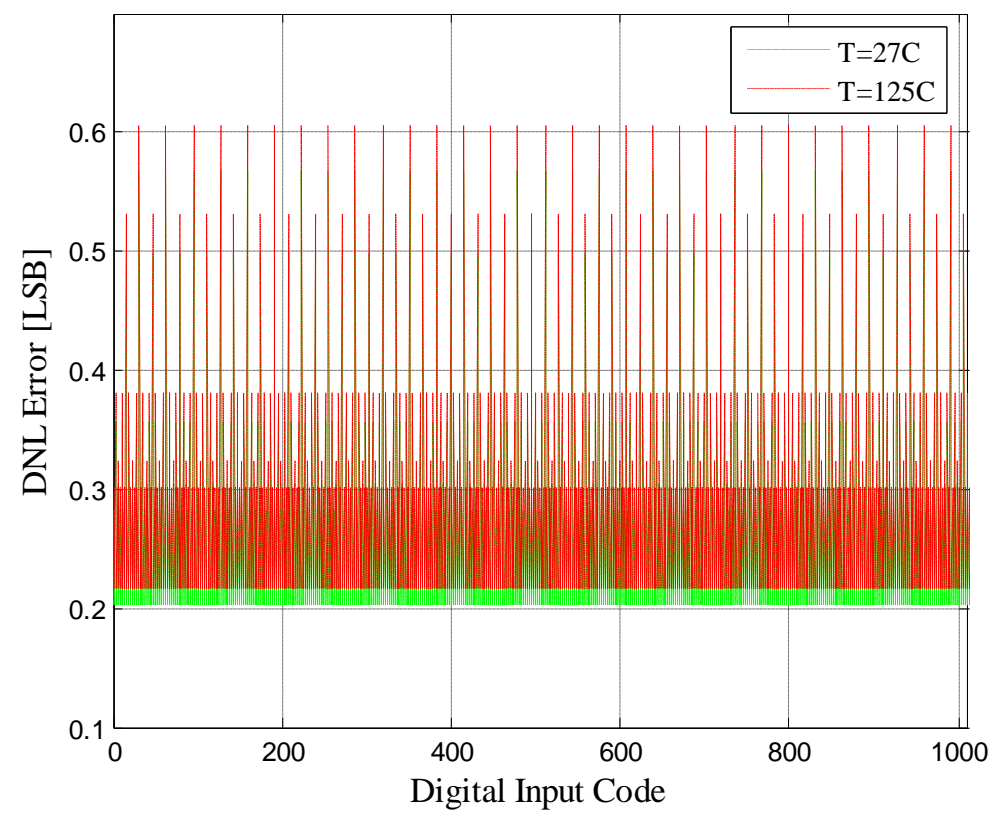

Figure 4.14 Simulated DNL characteristics of 10-bit gate-multiplied DAC using ideal bias voltage source at $27^{\circ} \mathrm{C}$ and $125^{\circ} \mathrm{C}$

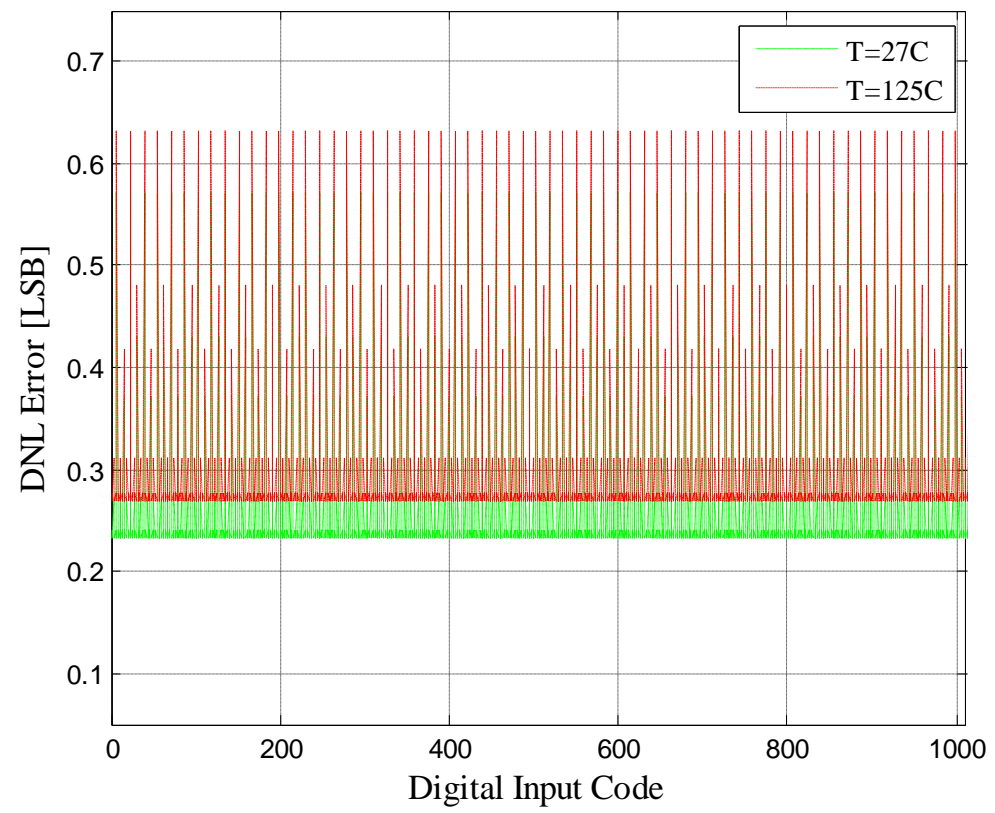

Figure 4.15 Simulated DNL characteristics of 10-bit width-customized DAC using ideal bias voltage source at $27^{\circ} \mathrm{C}$ and $125^{\circ} \mathrm{C}$ 


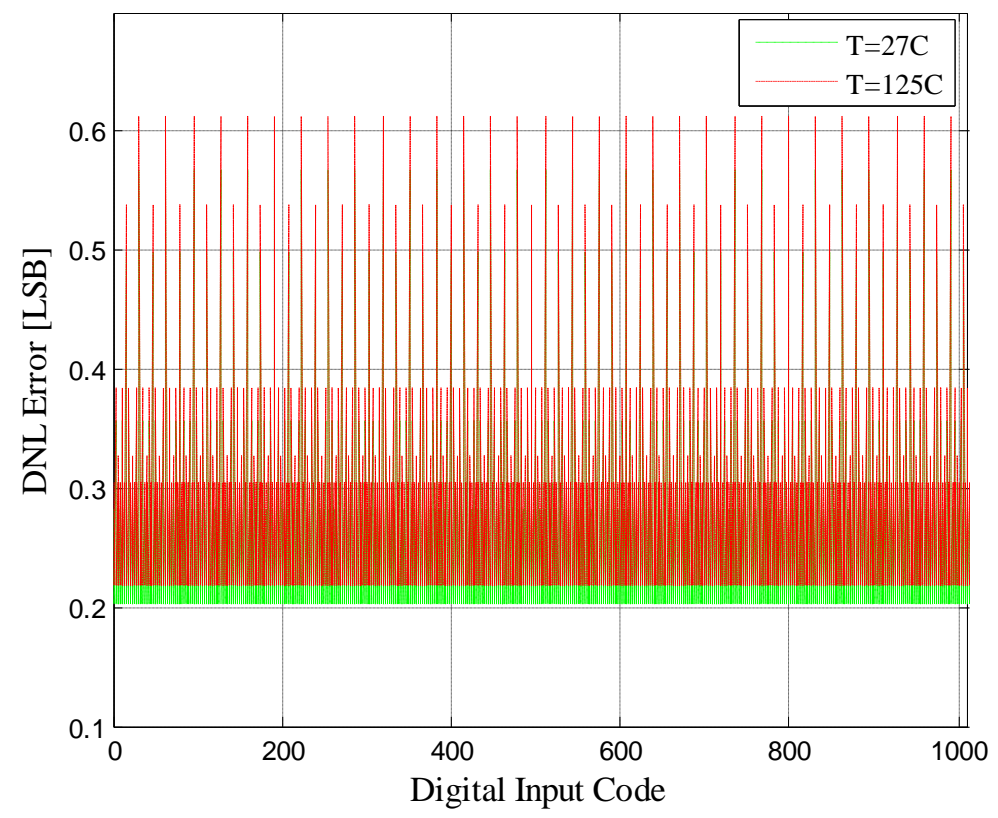

Figure 4.16 Simulated DNL characteristics of 10-bit gate-multiplied DAC using bias generation circuit at $27^{\circ} \mathrm{C}$ and $125^{\circ} \mathrm{C}$

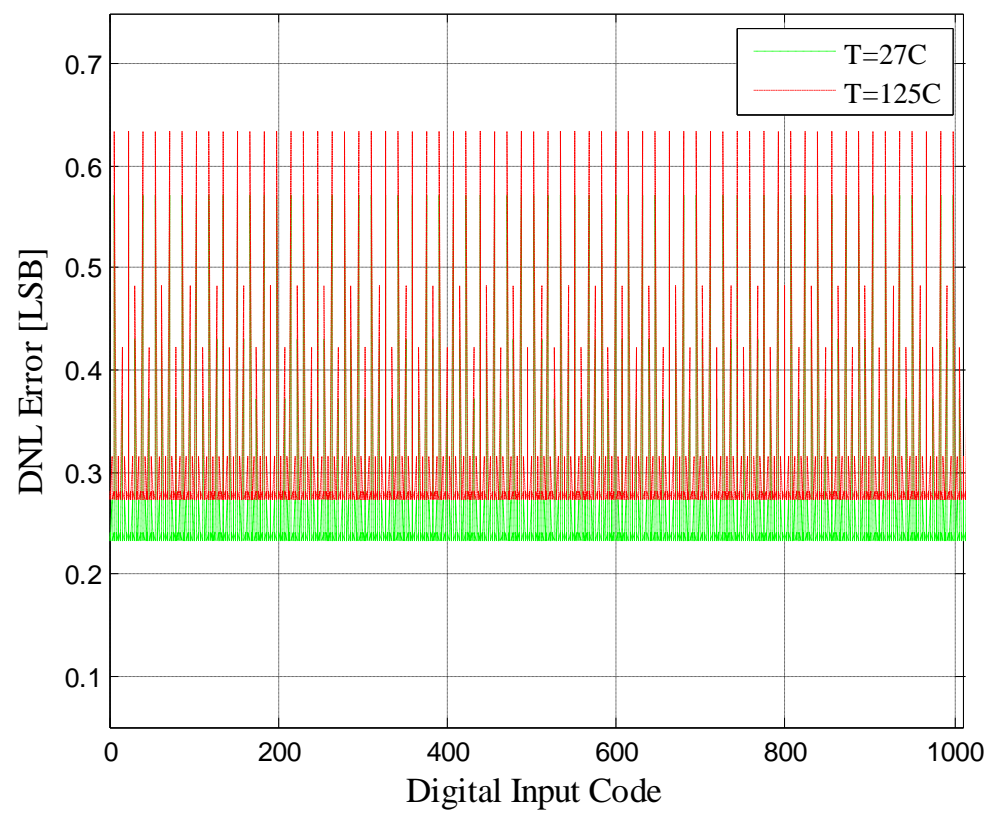

Figure 4.17 Simulated DNL characteristics of 10-bit width-customized DAC using bias generation circuit at $27^{\circ} \mathrm{C}$ and $125^{\circ} \mathrm{C}$ 


\section{CHAPTER V}

CONCLUSIONS

\subsection{Summary}

Two 10-bit current-mode MOSFET DACs are designed in 0.5- $\mu \mathrm{m}$ SOI technology: a gate-multiplied design with approximate die dimensions of $495 \mu \mathrm{m} \times$ $135 \mu \mathrm{m}$ and a width-customized design with approximate die dimensions of $150 \mu \mathrm{m} \times 92$ $\mu \mathrm{m}$. A new gate-controlled DAC architecture is implemented, and is found to overcome the non-linearity problems faced by the conventional drain-controlled DAC. Temperature-insensitive operation of the proposed DAC designs is achieved by operating the PMOS current sources at the ZTC voltage, when they are turned ON.

The transistor-level implementations of the proposed 10-bit DAC designs are simulated using all 1024 digital input combinations. Different performance measures of the DACs have been evaluated from the simulation results such as bit current ratios, INL and DNL, from which a comparison is drawn between the two proposed designs. From these results, it is observed that both the DAC designs (gate-controlled and widthcustomized) function with a maximum absolute error less than one LSB both at $27^{\circ} \mathrm{C}$ and slightly more than one LSB at $125^{\circ} \mathrm{C}$. Comparing the two designs, the gate-controlled DAC exhibits better performance than the width-customized DAC in terms of bit-current ratios, INL and DNL. 


\subsection{Future work}

The proposed design could be extended to any number of bits by adding more stages at the cost of increased area and power consumption. As a next step in the design flow, the layout of the proposed design can be sent to foundry for fabrication and can be tested for performance evaluation. This will help in analyzing the effect of process variations and mismatch errors, which occur during fabrication, on the performance of the proposed design.

The bias generation circuit used in this work to create the bias for the current sources is shown to have a variation of $0.9 \mathrm{mV}$ in the output voltage over a temperature range of $27^{\circ} \mathrm{C}$ to $125^{\circ} \mathrm{C}$. A more sophisticated bias generation circuit whose output voltage does not vary as much with the temperature can be developed.

A voltage-mode MOSFET DAC can be realized from the proposed current-mode MOSFET DAC by employing simple I-V conversion principle. This can be achieved using an op-amp with a feedback resistor. In this thesis, all the simulations were conducted with the sum node being held constant at zero potential, as would be the case when using an ideal op-amp for the I-V conversion. A preliminary simulation has been conducted to determine the error in the output current of the proposed DAC design with variations in the sum node voltage. The results showed that the sum of the errors in the ten bit currents would be less than the nominal LSB current provided that the sum node voltage was limited to variations no greater than $40.4 \mathrm{mV}$. An op-amp with relatively modest gain and accuracy specifications is capable of keeping the sum node variations within this limit. 


\section{BIBLIOGRAPHY}

[1] C.-H. Lin and K. Bult, "A 10-b 500-MSample/s CMOS DAC in 0.6 mm²," IEEE Journal of Solid-State Circuits, vol. 33, no. 12, December 1998.

[2] A. B. Grebene, "Bipolar and MOS Analog Integrated Circuit Design," WileyInterscience, 1984.

[3] K. L. Fong and C. A. T. Salama, "A 10 Bit Semi-Algorithmic Current Mode DAC," Proceedings of the International Symposium on Circuit and Systems, Chicago, Illinois, USA, 1993, pp. 978-981.

[4] T. Manku and Y. Wang, "Temperature-Independent Output Voltage Generated by Threshold Voltage of an NMOS Transistor," Electronics Letters, vol. 31, no. 6, pp. 935-936, 1995.

[5] I. M. Filanovsky and A. Allam, "Mutual Compensation of Mobility and Threshold Voltage Temperature Effects with Applications in CMOS circuits," IEEE Transactions on Circuits and Systems-I, vol. 48, no. 7, pp. 876-883, July 2001.

[6] B. Razavi, "Principles of Data Conversion System Design," IEEE Press, Piscataway, NJ, 1995.

[7] J.W. Bruce, "Nyquist-Rate Digital to Analog Converter Architectures," IEEE Potentials, vol. 20, no. 3, pp. 24-28, August 2001.

[8] D. Fitrio, J. Singh and A. Stojcevski, "Ultra Low Power Weak Inversion Current Steered Digital to Analog Converter," IEEE Asia Pacific Conference on Circuits and Systems, Macao, China, 2006, pp. 1545-1548.

[9] S. Sarkar, R. S. Prasad, S. K. Dey, V. Belde and S. Banerjee, "An 8-bit 1.8 V 500 MS/s CMOS DAC with a Novel Four-Stage Current Steering Architecture," International Symposium on Circuits and Systems, Seattle, Washington, USA, 2008, pp. $149-152$.

[10] C. Ionascu and D. Burdia, "Design and Implementation of Video DAC in 0.13/spl mu/m CMOS Technology," IEEE International Symposium on Signals, Circuits and Systems, Laui, Romania, July 2003,vol. 2, pp. 381-384. 
[11] J. Huynh, B. Ngo, M. Pham, L. He, "Design of a 10-bit TSMC 0.25um CMOS Digital to Analog Converter," Sixth International Symposium on Quality of Electronic Design, San Jose, CA, USA, 2005, pp. 187-192.

[12] F. Azais, S. Bernard, Y. Bertrand, and M. Renovell, "A Low-Cost BIST Architecture for Linear Histogram Testing of ADCs," Journal of Electronic Testing, vol. 17, pp. 139-147, 2001.

[13] T. Fleischmann and B. Mullane, "A Dynamic ADC Test Processor for Built-in Self-Test of ADCs," Design, Analysis and Tools for Integrated Circuits and Systems, Greece, 2008.

[14] T. J. Reilly, "Digital-to-Analog Converter With Temperature Compensation," United States Patent \#6,593,864. Assignee: Optical Solutions, Inc., Minneapolis, MN, 2003.

[15] S. Shah and S. Collins, "A temperature independent trimmable current source," International Symposium on Circuits and Systems, Scottsdale, AZ, USA, May 2002, vol. 1, pp. 713-716.

[16] N. Arora, MOSFET Modeling for VLSI Simulation: Theory and Practice, World Scientific Publishing Co. Pte. Ltd, Hackensack, NJ, 2007.

[17] S. Jeon and D. E. Burk, "A Temperature-Dependent SOI MOSFET Model for High-Temperature Application (27C-300C)," IEEE Transactions on Electron Devices, vol. 38, no. 9, September 1991. 